\title{
Statistical Significance of Periodicity and Log-Periodicity with Heavy-Tailed Correlated Noise
}

\author{
Wei-Xing Zhou 1 and Didier Sornette 늘.
}

1. Institute of Geophysics and Planetary Physics, University of California, Los Angeles, CA 90095

2. Department of Earth and Space Sciences, University of California, Los Angeles, CA 90095

3. Laboratoire de Physique de la Matière Condensée, CNRS UMR 6622 and Université de NiceSophia Antipolis, 06108 Nice Cedex 2, France

\begin{abstract}
We estimate the probability that random noise, of several plausible standard distributions, creates a false alarm that a periodicity (or log-periodicity) is found in a time series. The solution of this problem is already known for independent Gaussian distributed noise. We investigate more general situations with non-Gaussian correlated noises and present synthetic tests on the detectability and statistical significance of periodic components. A periodic component of a time series is usually detected by some sort of Fourier analysis. Here, we use the Lomb periodogram analysis which is suitable and outperforms Fourier transforms for unevenly sampled time series. We examine the false-alarm probability of the largest spectral peak of the Lomb periodogram in the presence of power-law distributed noises, of short-range and of long-range fractional-Gaussian noises. Increasing heavy-tailness (respectively correlations describing persistence) tends to decrease (respectively increase) the false-alarm probability of finding a large spurious Lomb peak. Increasing anti-persistence tends to decrease the false-alarm probability. We also study the interplay between heavy-tailness and long-range correlations. In order to fully determine if a Lomb peak signals a genuine rather than a spurious periodicity, one should in principle characterize the Lomb peak height, its width and its relations to other peaks in the complete spectrum. As a step towards this full characterization, we construct the jointdistribution of the frequency position (relative to other peaks) and of the height of the highest peak of the power spectrum. We also provide the distributions of the ratio of the second highest Lomb peak to the maximum peak. Using the insight obtained by the present statistical study, we re-examine previously reported claims of "log-periodicity" and find that the credibility for logperiodicity in 2D-freely decaying turbulence is weakened while it is strengthened for fracture, for the ion-signature prior to the Kobe earthquake and for financial markets.
\end{abstract}

\section{Introduction}

The problem of detecting oscillatory components in noisy data is one of the most general problems found in all scientific disciplines. Without being exhaustive, the search of periodic signals in complex time series goes from astrophysics (for instance in the detection of planets outside our solar system), geosciences (for instance in the detection of cycles in meteorology and climate dynamics), medicine (for instance in the effect of circadian cycles), to economics (for instance in the business cycles or in the calendar effects occurring at intra-day, week, month and year time scales in financial times series). It is not stretching reality to state that almost all fields have at least one outstanding 
question related to the detection of tiny periodic oscillations in an otherwise noisy and complex background. Many techniques have been invented, adapted or improved to address the specificities of each situation.

The most standard tool for detecting a periodic component in a noisy time series is the Fourier transform (FT) and, when possible, the fast Fourier transform (FFT).

The FT and FFT methods require evenly sampled signals. However, due to measurement constraints, it can happen that the time series is not evenly spaced in time. A straightforward approach is to reconstruct evenly spaced data using interpolation or rebinning and then apply the FFT method. However, interpolation and rebinning both introduce some distortion and may lose some information in situations where the periodic signal is weak and difficult to distinguish from noise.

In addition, the FT and FFT methods perform well only when the the data size is relatively large so as to minimize aliasing (window border) distortions at the extremities of the time series. Wavelet techniques and singular spectral analysis have been developed to address in part this problem.

A completely different method for detecting periodic components in an unevenly sampled and relatively short signal is the Lomb periodogram [1]. The Lomb periodogram analysis performs local least-squares fit of the data by sinusoids centered on each data point of the time series [2]. Given a unevenly spaced signal $y\left(t_{i}\right)$, where $i=1,2, \cdots, N$, the normalized Lomb periodogram is calculated according to the following formula [2, 3, 1]:

$$
P_{N}(\omega)=\frac{1}{2 \sigma^{2}}\left[\frac{\left[\sum_{i=1}^{N} y\left(t_{i}\right) \cos \omega\left(t_{i}-\tau\right)\right]^{2}}{\sum_{i=1}^{N} \cos ^{2} \omega\left(t_{i}-\tau\right)}+\frac{\left[\sum_{i=1}^{N} y\left(t_{i}\right) \sin \omega\left(t_{i}-\tau\right)\right]^{2}}{\sum_{i=1}^{N} \sin ^{2} \omega\left(t_{i}-\tau\right)}\right],
$$

where $\tau$ is defined by the equation

$$
\tan (2 \omega \tau)=\left(\sum_{i=1}^{N} \sin 2 \omega t_{i}\right) /\left(\sum_{i=1}^{N} \cos 2 \omega t_{i}\right)
$$

and $\sigma^{2}$ is the total variance of the data (including noise and signal) odogram $P_{N}(\omega)$ is similar to a FT power spectrum in which the presence of peaks at certain angular frequencies $w_{n}$ signals the possible existence of periodic components. The largest peaks point to the most important frequencies in the time series, the higher is a peak, the larger is the statistical significance of the corresponding periodic component. Due to its local least-square fit nature, the Lomb periodogram analysis works equally well for unevenly spaced time series. It is also much less prone to aliasing (window border) distortions in short time series.

To understand intuitively how the expression (11) allows one to detect periodic components, let us consider a noisy signal $y(t)$ made of a finite number of independent periodic functions plus a noise $x(t)$ of zero mean which is independent of the periodic components:

$$
y(t)=\sum_{m=1}^{M} y_{m} \cos \left(\omega_{m} t\right)+x(t) .
$$

Using the independence between the noise and the periodic signals, we obtain $\langle y\rangle=0$ and $\sigma^{2}=$ $\left\langle y^{2}\right\rangle=\sum_{1}^{M} y_{m}^{2} / 2+\sigma_{x}^{2}$. Since the translation $\tau$ in Eq. (1) provides usually a very minor correction, we can ignore it here. In addition, we assume that the $t_{i}$ 's are not badly bunched [3]. Given these two conditions, the Lomb periodogram at angular frequency $\omega_{m}$ can be written as

$$
P_{N}\left(\omega_{m}\right)=\frac{N}{2}\left(\frac{\sum_{1}^{M} y_{m}^{2}}{y_{m}^{2}}+\frac{2 \sigma_{x}^{2}}{y_{m}^{2}}\right)^{-1} .
$$

\footnotetext{
${ }^{1}$ The formula (1) uses the total variance of the data which is generally available rather than the variance of the noise derived either from the residuals after subtracting a signal or from the uncertainty of the measurements [3].
} 
This result (丹) emphasizes the importance of normalizing the periodogram by the total variance of the data. It also shows that the Lomb peak height is approximately proportional to the number $N$ of points and to the square of the the amplitude $y_{m}$ of a given periodic component. Note that Eq. (4) holds approximately for all types of noises, not only for Gaussian noise. Numerical verifications for the case of a single noisy sine signal, i.e. $M=1$, have been presented in Ref. [3].

A severe drawback when using Eq. (11) directly is computational: the time needed to calculate a Lomb periodogram typically grows as $N^{2}$ compared to $N \ln N$ for the FFT. To fix this, a fast computation approach of the Lomb periodogram has been proposed [ [ 7 , 1]. The method uses the FFT, but it is not a FFT periodogram of the data. In this method, $P_{N}(\omega)$ can be calculated with any desired precision and the time needed is only of order $N \ln N$. This is the method used in the present work.

However, the presence of a peak in the Lomb periodogram does not guarantee the presence of a genuine periodic signal as random fluctuations can create peaks by chance alone. What is needed is a quantitative assessment of the probability that peaks can be generated by random fluctuations. The statistical significance of a given peak is the probability that it does not stem from noise. In this goal, previous works [1] have defined a null hypothesis, which assumes that a given peak observed in the Lomb periodogram stems from independent Gaussian noise. Here, we shall develop a hierarchy of more sophisticated null hypothesis.

If the signal $y\left(t_{i}\right)$ is a pure noise, namely the noise is independently normally distributed then, at any particular $\omega$, it can be shown that the normalized periodogram $P_{N}(\omega)$ has an exponential probability distribution with unit mean [2]. In other words, the probability that the height $z=$ $P_{N}(\omega)$ of the Lomb periodogram at any given frequency is found between some positive $z$ and $z+d z$ is $e^{-z} d z$ [B], independently of the amplitude of the Gaussian noise. This independence with respect to the noise amplitude stems from the normalization explicited in equations (11.4). If we scan some $M$ independent frequencies $\omega_{1}, \omega_{2}, \ldots, \omega_{M}$, the probability that none of these $M$ frequencies give a corresponding value of the Lomb periodogram $P_{N}\left(\omega_{1}\right), P_{N}\left(\omega_{2}\right), \ldots, P_{N}\left(\omega_{M}\right)$ larger than $z$ is $\left(1-\mathrm{e}^{-z}\right)^{M}$. Therefore, the probability for any Lomb peak to exceed $z$ is

$$
\operatorname{Pr}(>z)=1-\left(1-e^{-z}\right)^{M}
$$

which defines the false-alarm probability of the null hypothesis. The significance level of any peak $P_{N}(\omega)$ is thus $1-\operatorname{Pr}(>z)$. A small value for the false-alarm probability indicates a highly significant periodic signal. The interesting region is where the false-alarm probability is small such that equation (5) can be expanded to give

$$
\operatorname{Pr}(>z) \approx M e^{-z}
$$

In order to estimate the false-alarm probability, extensive Monte Carlo simulations have been carried out to determine the values of $M$ in various cases [3]. In those simulations [3], all data sets were Gaussian noise with different spacings in time: (1) evenly spaced data in time; (2) time increments drawn as uniformly distributed random numbers in $(0,1)$; and (3) increments clumped in groups. These works [2, 3] thus aimed at testing the impact of different non-uniform sampling rates, rather than the effect of non-Gaussian non-white noise as studied here.

Our interest in this problem is motivated by the problem of detecting so-called log-periodic oscillations in self-similar systems, which are the signatures of the symmetry of discrete scale invariance (DSI) [5]. The hallmark of self-similarity is the presence of a power law followed by an observable $F(x)$ as a function of the scale $x$ of observation or of the distance $x$ to the critical point. The hallmark of DSI is the existence of oscillations that are periodic in the logarithm of $x$, hence 
the name "log-periodic". As a complement to fitting an observable with a power law decorated by $\log$-periodic oscillations, a non-parametric detection of log-periodicity is of great interest to test for the presence of DSI and was introduced in [6, 7, 8] based on the Lomb periodogram.

Such a method indeed requires the use of the Lomb periodogram analysis because, by construction, the sampling is non-uniform since the analysis must be performed in the variable $\ln x$. In addition, only a few log-periodic oscillations are usually available, since each fully completed oscillation corresponds to a multiplication of the observable by a preferred scaling ratio $\lambda>1$. Hence, $n$ oscillations correspond to spanning the interval $\left[x_{0}, x_{0} \lambda^{n}\right]$, where $x_{0}$ is some constant. Thus many decades of data are required to observe only a few oscillations. In order to perform the Lomb periodogram of an observable exhibiting scale invariance, one can either subtract a pure power law fit from the data and analyze the residue with the Lomb periodogram method in terms of the variable $\ln x[9,10]$ or define a local exponent by constructing a local logarithmic derivative $d \ln F / d \ln x$. Then, the potential oscillatory structure of the fluctuations of this local exponent as a function of $\ln x$ can be analyzed [11].

The quantification of the false-alarm probability given by (5) and (5) is based on the assumption that the noise is Gaussian and uncorrelated, as mentioned above. In practice, this is almost always wrong. The present work investigates how the false-alarm probability $\operatorname{Pr}(>z)$ (and therefore the confidence level $1-\operatorname{Pr}(>z)$ ) changes from the predictions (5) and (6) in the presence of nonGaussian noise and of dependence between successive innovations (increments) of the time series. If one knows some of the characteristics of the noise distribution and of its dependence structure, a more refined null hypothesis asks the following question: what is the false-alarm probability of the highest peak of a Lomb periodogram performed on a pure noise with the same distribution and same dependence structure?

Two recent papers [9, 10] have already partially addressed this question. Ref. [9] identified a novel source for periodicity relying solely on the manipulation of data: (1) approximate uniform sampling and (2) use of cumulative or integrated quantities which reddens the noise (increases the power spectrum at low frequencies) and, in a finite sample, creates a maximum in the spectrum leading to a most probable frequency. It was found that (i) the frequencies of the oscillations have a power spectrum given approximately by $\omega^{-2}$; (ii) the most probable frequency scales as the inverse of the interval size; (iii) the amplitude of the periodic oscillations scales as the inverse square root of the number of data points (central limit theorem). These properties were studied in the context of the search for log-periodicity in earthquake aftershock time series, where the natural variable is $\ln t$. Ref. [10] presented a battery of synthetic tests performed to quantify the statistical significance of the existence of log-periodic oscillations in cumulative seismic data up to major earthquakes. By defining synthetic tests that are as much as possible identical to the real time series except for the property to be tested, namely log-periodicity, Ref. [10] found that log-periodic oscillations with frequency and regularity similar to those of the real seismic data are likely to be generated by the interplay of the low pass filtering step due to the construction of cumulative functions together with an approximate power law acceleration.

As a prerequisite of the analysis of log-periodicity in many systems (growth processes, rupture, earthquakes, turbulence, finance, etc), it is necessary to extend the previous investigation on the Lomb analysis to non-Gaussian noise with the possible addition of correlated noise. In the sequel, we examine the following three cases: 1) noises with heavy tails but no correlations, 2) Gaussian noises with correlations, and 3) noises with both non-Gaussian heavy tails and correlations. We perform extensive numerical simulations to determine the effects of either heavy-tailness or correlations on the significance level of the peaks.

Our presentation is organized as follows. We investigate the effect of heavy-tailness in noises 
on the significance level of the signals in Sec. 2. We analyze two types of noises, the first class characterized by Lévy stable distributions and the second one distributed according to general power law distributions. In Sec. 3, we consider fractal-Gaussian noises with long-range corrections (both persistent and anti-persistent). The interplay between heavy-tailness and correlations in noises is studied in Sec. 月. Together with the heavy-tailed noise distributions, we consider both short-range exponentially decaying correlations as well as very long-range correlation such as in fractional Gaussian motions. We consider the joint distribution of the frequency and height of the highest peak of the Lomb power spectrum in Sec. 5. Application of the numerical results to real systems are discussed in Sec. 6. We summarize our results in Sec. П].

\section{Effect of heavy-tailness}

\subsection{Lévy stable noise}

We consider an uncorrelated Lévy noise [12], in which each innovation (increment) is independently drawn from the same Lévy distribution. There is no closed analytical expression for the probability function of the stable distributions with a few exceptions, e.g., Cauchy distribution $(\alpha=1)$ and Gaussian distribution $(\alpha=2)$ [13]. Stable distributions are given in terms of their characteristic function [14]. We denote $X \sim B^{\alpha}(\lambda, \mu, \sigma)$ or $B^{\alpha}(x ; \lambda, \mu, \sigma)$ such a Lévy stable distribution. The symbol $\sim$ means that the random variable $X$ is drawn from the process $B^{\alpha}(\lambda, \mu, \sigma)$. This process is specified by four parameters: $\alpha$ is the stable characteristic exponent $(0<\alpha \leq 2), \lambda$ is the symmetry parameter $(-1 \leq \lambda \leq 1), \sigma$ is the scale factor $(\sigma>0)$, and $\mu$ is the location parameter. When $\alpha=2$, the distribution is Gaussian with mean $\mu$ and variance $2 \sigma^{2}$, and $\lambda$ has no effect. The tail probability distribution in the non-Gaussian cases $\alpha<2$ is known asymptotically as [14, 15]:

$$
\operatorname{Pr}\left(B^{\alpha}>x\right) \simeq \sigma^{\alpha} \frac{1+\lambda}{2} C_{\alpha} x^{-\alpha},
$$

where $C_{\alpha}=\frac{1-\alpha}{\Gamma(2-\alpha) \cos (\pi \alpha / 2)}$, if $\alpha \neq 1$. Hence, stable distributions have heavy tails: the lower the value $\alpha$ is, the more extended the tails become and the greater is the probability of extreme events. In the case of $\lambda=0$, we have the expression of symmetric Lévy stable distributions [16, 17]:

$$
f(x)=\frac{1}{\pi} \int_{0}^{\infty} \exp \left(-\sigma q^{\alpha}\right) \cos (q x) d q .
$$

Our generation algorithm is based on the so-called CMS method [18], which is applied in such a way that $x$ will have the characteristic function $\Phi(\theta)$ of the Lévy stable distribution [14]. With this parameterization, the stable distribution $B^{\alpha}(x ; \lambda, \mu, \sigma)$ equals $B^{\alpha}\left(\frac{x-\mu}{\sigma} ; \lambda, 0,1\right)$ [19]. We used the program provided by McCulloch 2. In our simulations, we fixed $\lambda=0, \mu=0$ and $\sigma=\frac{1}{\sqrt{2}}$, and changed $\alpha$ from 0.1 up to 2 with step 0.1 so that we can compare our results with those of Gaussian white noise. Four typical noises with 10,000 data points are illustrated in Fig. 1: (a) $\alpha=2$, (b) $\alpha=1.5$, (c) $\alpha=1$, and (d) $\alpha=0.5$. For each $\alpha$, we generated 50,000 data sets each with 100 data points. The unevenly spaced sampling of times which is adopted throughout this paper is generated as follows: one first generates 100 uniformly distributed random numbers between 0 and 1, and then obtains the cumulative sums that are used as the sampling times $t_{1}, \cdots, t_{100}$. By construction, the sampling intervals $\Delta t=t_{i+1}-t_{i}$ are uniformly distributed. We performed the Lomb analysis on each data set.

\footnotetext{
${ }^{2}$ Available at http://economics.sbs.ohio-state.edu/jhm/jhm.htm
} 
Fig. 2 2 shows the four Lomb periodograms corresponding to the four time series shown in Fig. 1. It is visually suggesting that the spontaneous emergence of peaks above the background becomes less and less probable for smaller $\alpha$. This is confirmed by extensive statistical tests synthetized in Fig. 3 which plots the false-alarm probability as a function of the Lomb peak for different exponents $0.1 \leq \alpha \leq 2$. For reference and comparison, the Gaussian case is represented by the open circles. Within statistical fluctuations, it is identical to the last curve to the right corresponding to $\alpha=2$, as it should. Remarkably, for a given Lomb peak height, the false-alarm probability increases with $\alpha$. Hence, a Lévy noise with heavier tail (smaller exponent $\alpha$ ) results in a higher significance for a given Lomb peak height. In other words, it is more difficult to generate a large peak in the Lomb periodogram analysis when the noise has more extended tail. Intuitively, the larger amplitudes of the noise fluctuations increase the effective randomness of the noise and destroy possible oscillations constructed by random occurrences. In other words, Fig. 11 suggests that, the smaller $\alpha$ is, the smaller is the number of points dominating the overall signal: for instance the last panel for $\alpha=$ 0.5 shows that only a few very large peaks dwarf the rest of the population of noise innovations (increments). Since there is very little structure, there is little chance for a periodic component to appear, hence the small false alarm probability.

The semi-logarithmic representation of Fig. 3 suggests that the false-alarm probability is approximately an exponentially decaying function of the Lomb peak, similarly to the Gaussian case described by Eq. (6). This suggests to fit the dependence of the false-alarm probability for various $\alpha$ as an exponential of the Lomb peak height

$$
\operatorname{Pr}_{\alpha}(z)=M(\alpha) e^{-k(\alpha) z},
$$

with an effective number $M(\alpha)$ of independent frequencies and a decay rate $k(\alpha)$, which are expected to be respectively increasing and decreasing functions of $\alpha$.

In order to obtain more reliable estimations of $M(\alpha)$ and $k(\alpha)$, we excluded the largest $z$ 's from the fit with Eq. (9), because they are spoiled by large fluctuations. The scaling range was fixed in the interval $\operatorname{Pr}_{\alpha}(z) \in[0.001,0.05]$. The upper bound 0.05 ensures the validity of the approximation (6). The lower bound 0.001 is a good compromise to have both a large range for the fit and to exclude the largest fluctuations occurring for the smallest $\operatorname{Pr}_{\alpha}(z)$. The error bars for $k(\alpha)$ are estimated by evaluating the standard deviation over 12 subintervals constituted of the ten subintervals resulting from subdividing the range $[0.001,0.05]$ equally in ten parts and adding two adjoining subintervals of the same size, one below the lower bound and one above the upper bound. The results are listed in Table 1. We find $M(2)=104$ and $k(2)=1.14 \pm 0.19$, which are consistent with the results $M=119$ and $k=1$ known for independent Gaussian noise [3]. Table 11 shows a systematic decrease of $k(\alpha)$ as $\alpha$ increases, which is capturing the observation that the false-alarm probability is increasing with $\alpha$. We find however that the determination of the effective number $M(\alpha)$ of independent frequencies is not reliable as its determination is very sensitive to the value $k(\alpha)$. Therefore, we cannot conclude about a specific dependence of $M(\alpha)$ as a function of $\alpha$. This limitation is relatively minor as this pre-exponential factor $M(\alpha)$ has a weak impact on the false-alarm probability (a factor two or three at most).

\subsection{Symmetrical power-law noises}

We now consider noises distributed with density distribution functions decaying as pure power laws according to

$$
f(y)=\left\{\begin{array}{cc}
\frac{\kappa}{2|y|^{\kappa+1},}, & |y|>1 \\
0, & |y| \leq 1
\end{array}\right.
$$




\begin{tabular}{|c|c|c|c|c|c|}
\hline$\alpha$ & 0.1 & 0.2 & 0.3 & 0.4 & 0.5 \\
\hline$k$ & $3.06 \pm 0.72$ & $2.89 \pm 0.74$ & $2.69 \pm 0.40$ & $2.42 \pm 0.41$ & $2.12 \pm 0.25$ \\
\hline$M$ & 32 & 90 & 154 & 151 & 90 \\
\hline$\alpha$ & 0.6 & 0.7 & 0.8 & 0.9 & 1.0 \\
\hline$k$ & $1.98 \pm 0.27$ & $2.04 \pm 0.29$ & $1.89 \pm 0.36$ & $1.73 \pm 0.24$ & $1.59 \pm 0.18$ \\
\hline$M$ & 97 & 224 & 185 & 133 & 92 \\
\hline$\alpha$ & 1.1 & 1.2 & 1.3 & 1.4 & 1.5 \\
\hline$k$ & $1.59 \pm 0.24$ & $1.50 \pm 0.33$ & $1.49 \pm 0.15$ & $1.39 \pm 0.17$ & $1.33 \pm 0.23$ \\
\hline$M$ & 150 & 121 & 166 & 131 & 112 \\
\hline$\alpha$ & 1.6 & 1.7 & 1.8 & 1.9 & 2.0 \\
\hline$k$ & $1.27 \pm 0.11$ & $1.19 \pm 0.15$ & $1.16 \pm 0.12$ & $1.12 \pm 0.20$ & $1.14 \pm 0.19$ \\
\hline$M$ & 101 & 78 & 85 & 80 & 104 \\
\hline
\end{tabular}

Table 1: List of the parameters $k(\alpha)$ and $M(\alpha)$ of the fit of the false-alarm probability $\operatorname{Pr}_{\alpha}(z)$ by expression (9).

These distributions complement the results obtained using the Lévy stable distributions of the previous section by 1) testing for the role of a strong difference in the bulk part of the distributions and 2) allowing for the exponent $\kappa$ to be larger than 2 .

We first generate uniformly distributed random numbers $x$ in $(0,1)$, and then substitute them into

$$
y=\left\{\begin{array}{cc}
-(2 x)^{-1 / \kappa}, & 0<x \leq 0.5, \\
{[2(1-x)]^{-1 / \kappa},} & 0.5<x<1 .
\end{array}\right.
$$

In the simulations, we consider values of $\kappa$ from 0.1 to 2 with spacing 0.1 , from 2.5 to 5 with spacing 0.5 , and the values $\kappa=6$ and $\kappa=7$. Four typical noises with 10,000 data points are illustrated in Fig. 4: (a) $\kappa=5$, (b) $\kappa=2$, (c) $\kappa=1.5$, and (d) $\kappa=0.5$. For each $\kappa$, we generated 50,000 data sets, each with 100 data points, as in Sec. 2.1. Figure 5 plots the false-alarm probability as a function of the Lomb peak for different exponents $0.1 \leq \kappa \leq 7$. For reference and comparison, the Gaussian case is represented by the open circles. The results are very similar to those found for the Lévy noise shown in Fig. 3. As the exponent $\kappa$ increases, the false-alarm probability increases: the thinner the tail is, the smaller is the confidence level of a given Lomb peak. Intuitively, the results are as for the Lévy noise: largest fluctuations for small $\kappa$ introduce a larger effective randomness making less probable the occurrence of oscillations. Similarly to the argument proposed in the previous section, Fig. 7 suggests that, the smaller $\kappa$ is, the smaller is the number of points dominating the overall signal. As a consequence, the time series will have little structure, hence the small false-alarm probability.

In contrast with the Lévy noise for which the false-alarm curves accumulate at the Gaussian curve for $\alpha=2$, the false-alarm curves for the power law noises continue to translate to the right as $\kappa$ increases. The reason is that $\kappa=2$ for a power law distribution is not the same as $\alpha=2$ for a Lévy stable distribution: the former case corresponds to a power law distribution $\sim|y|^{-3}$ while the latter corresponds to the Gaussian distribution. Interestingly, for $\kappa>3$, we observe that the false-alarm probabilities are larger than for the Gaussian case. In this sense, uncorrelated noises with power law distributions with tail exponent $\kappa>3$ are "less random" than the Gaussian case as oscillations caused by random occurrences are more frequent. 
The reason why a more extended tail leads on average to smaller Lomb peaks, such that a given peak height has stronger false-alarm probability (smaller statistical significance) for the Gaussian noise than for Lévy noise, can be easily understood from the following argument. Qualitatively, a smaller exponent leads to larger fluctuations which introduce a larger effective randomness making less probable the occurrence of oscillations. Quantitatively, this "larger randomness" can be measured by the sample-size-dependence of the empirical variance $\sigma^{2}$ entering the height of the Lomb peak according to expression ( $(4)$. Let us present our argument for the power laws (10) for which the explicit dependence on the exponent is simpler. Here, we use the same notation $\alpha=\kappa$ to stress that the argument applies in general to distributions with power law tails. Consider the case $\alpha<2$ for power laws for which the variance does not exist from a mathematical point of view. Notwithstanding this fact, one can always calculate an empirical variance from each specific time series, as

$$
\tilde{\sigma}^{2}=\frac{1}{N-1} \sum_{i=1}^{N}\left(x_{i}-\left\langle x_{i}\right\rangle\right)^{2},
$$

where $x_{i}$ is the $i$ th point of the synthesized Lévy stable noise. The notation $\left\langle x_{i}\right\rangle$ refers also to the empirical estimation of an average from the empirical time series. The fact that the variance (and the mean for $\alpha \leq 1$ ) does not exist is reflected in the fact that the estimated variance grows with data size $N$ and exhibits large fluctuations. $\tilde{\sigma}^{2}$ is scaling as

$$
\tilde{\sigma}^{2} \propto N \alpha \int_{1}^{x_{\max }} d x \frac{x^{2}}{x^{1+\alpha}} \propto \frac{N \alpha}{2-\alpha}\left(N^{\frac{2-\alpha}{\alpha}}-1\right),
$$

where we have estimated the typical largest value $x_{\max }$ by the standard condition

$$
N \alpha \int_{x_{\max }}^{\infty} d x x^{-1-\alpha}=1
$$

leading to the estimation $x_{\max }=N^{1 / \alpha}$. Expression (14) expresses the fact that the product of the probability to be of the order of or larger than $x_{\max }$ by the total number of points is of the order 1 , which defines $x_{\max }$. Figure 6 shows the maximal Lomb peak $P_{N}(\omega)$ averaged over 50,000 realizations of 100 points as a function of $\tilde{\sigma}^{2}$ given by expression (13). Here $\tilde{\sigma}^{2}$ is varied by changing the power law exponent from $\alpha=0.1$ to 1.9 . Increasing $\alpha$ decreases $\tilde{\sigma}^{2}$ and thus corresponds to reading figure 6 from right to left. Figure 7 shows directly the dependence of $P_{N}(\omega)$ as a function of $0.1 \leq \alpha \leq 1.9$ in the same range. This rationalizes our empirical finding that the Lomb peak $P_{N}(\omega)$ and as well as the maximal peak increases with $\alpha$. This is in line with the results presented in Figs. 3 and 5 . We will observe a similar effect in $\operatorname{GARCH}(1,1)$ noise discussed in Sec.4.1.

\section{Effect of correlations}

\subsection{Fractional Gaussian noises}

We now study the effect of correlations included in fractional Gaussian noise (fGn) $X_{H}(t)$ of parameter $H$. The cumulative sum $B_{H}(t)$ of fGn defines the fractional Brownian motion (fBm) which was introduced by Mandelbrot and Van Ness [20] as an extension to the usual Brownian motion. A process $B_{H}(t)$ is a $\mathrm{fBm}$ if it is a Gaussian random process which has stationary and self-similar increments,

$$
X_{H}(t)=B_{H}(t+1)-B_{H}(t)
$$


which form a fractional Gaussian noise (fGn). The value $H=\frac{1}{2}$ yields the ordinary Brownian motion, which is known to be non-persistent with absence of correlations of the increments $X_{1 / 2}(t)$. For $0<H<\frac{1}{2}$, the $\mathrm{fBm}$ is antipersistent, while for $\frac{1}{2}<H<1$, the $\mathrm{fBm}$ is persistent [21].

There are numerous synthesis methods proposed to generate $\mathrm{fBm}$, and hence fGn. They include the random midpoint displacement method [21, 22, 23], the Cholesky decomposition method of covariance matrix of vector increments using the Durbin-Levinson algorithm [24, 25, 26], the inverse Fourier transform method based on power spectrum [27, 28], the fast Fourier transform method matching covariance structure instead of frequency spectrum [29, 30], more recently the wavelet transform method [31, 32, 33, 34] and others [35, 30].

We adopted the Cholesky-Levinson factorization method[3, which is one of the best methods among those used to synthesize Gaussian time series [35]. This algorithm for generating fBm performs a linear transformation of i.i.d. Gaussian random variables in three steps: generation of independent Gaussian random numbers, multiplication of these random numbers by weights that are recursively determined by the covariance structure, and construction of their running sum [25]. The fGn are the innovations (increments) of $\mathrm{fBm}$. In the sequel, we analyze the statistical significance of the Lomb periodogram of fGn.

\subsection{Numerical results}

In our simulations, the Hurst index ranges from 0.1 to 0.9 with spacing 0.1 . For each $H$, we generated 50,000 fGn time series. The length of each time series is again 100 data points. Figure 8 shows four fractional Gaussian noises: (a) $H=0.2$, (b) $H=0.5$, (c) $H=0.7$, and (d) $H=0.9$. It is visually apparent that, the larger is $H$, the clearer is the presence of oscillations within the noise.

We performed the Lomb analysis on each fGn time series and extracted the largest peaks in the Lomb periodograms. The dependence of the false-alarm or rejection probability as a function of the Lomb peak height for different types of noises is shown in Fig. 9. For $H=0.5$, we recover the exponential decay (6) represented by the open circles. As $H$ increases, the false-alarm probability increases very significantly and also exhibits important deviations from an exponential fall-off.

For $H<0.5$, the fGn is anti-persistent. Thus, a point with negative (resp. positive) value in the series is usually followed by a point with positive (resp. negative). This effect favors a random oscillatory pattern at the highest possible frequency corresponding to a period of two points but the number of independent frequency is thus small. For smaller frequencies, this antipersistence makes improbable the spontaneous formation of other oscillatory regularities. In contrast, the fGn with $H>0.5$ is persistent. Thus a point of negative (resp. positive) value in the series is usually followed by a point of negative (resp. positive) value. Hence, there are more regularities and structure than for the Gaussian white noise. Long-range correlations create strong artifactual periodicity. This recovers while generalizing to different noise spectra the results of [9, 10]. A larger $H$ implying a larger correlation between the successive data points, and hence stronger regularities, it is natural to obtain a higher Lomb peak. This is true for the power spectra of the fGns proportional to $1 / f^{\beta}$ with $\beta=2 H-1<2$ which corresponds to long-range correlated stationary noises analyzed here. This is also true for noise spectral with exponent $\beta \geq 2$ for which the processes are not stationary (as in a random walk whose standard deviation grows as $\sqrt{t}$ ) with correlation functions blowing up.

\footnotetext{
${ }^{3}$ We used the MATLAB script named fbmlevinson.m included in FracLab available at http://wwwrocq.inria.fr/fractales/Software/FRACLAB/.
} 


\section{Interplay between heavy tail and correlations}

\subsection{The GARCH(1,1) process}

\subsubsection{Method for generation of $\operatorname{GARCH}(1,1)$ residuals}

Autoregressive Conditional Heteroscedasticity $(\mathrm{ARCH})$ process was introduced by Engle in 1982 [36] to account for the so-called heteroscedasticity in economic time series. Heteroscedasticity stands for the lack of stationary "volatility" (which is the term for standard deviation using in finance), i.e., the presence of periods of time with large volatilities alternating with periods of small volatility. In an $\operatorname{ARCH}(q)$ process the volatility at time $t$ is a function of the observed data at $t-1$, $t-1, \cdots, t-q$. In 1986, Bollersev [37] introduced the Generalized $\operatorname{ARCH}$ or $\operatorname{GARCH}(p, q)$ process, where the volatility at time $t$ depends on the observed data at $t-1, t-1, \cdots, t-q$, as well as on volatilities at $t-1, t-1, \cdots, t-p$. Here, we focus our attention on one of the standard benchmark model of financial time series, the $\operatorname{GARCH}(1,1)$ process, which obeys the following evolution equation:

$$
\begin{gathered}
x_{t}=\mu+\epsilon_{t}, \\
\epsilon_{t}=\sqrt{h_{t}} z_{t}, \\
h_{t}=\alpha+\beta \epsilon_{t-1}^{2}+\gamma h_{t-1},
\end{gathered}
$$

where $z_{t}$ is a standardized, independent, identically distributed (i.i.d.) random variable drawn from some specified probability distribution:

$$
\left\langle z_{t}\right\rangle=0 \quad \text { and } \quad\left\langle z_{t}^{2}\right\rangle=1 .
$$

The GARCH models have been shown to capture not only volatility clustering but also accommodate some of the leptokurtosis (i.e., heavy tails) commonly found in stock market and currency exchange rate time. However, GARCH models with conditionally normal errors generally fail to sufficiently capture the leptokurtosis evident in asset returns. The increasing attention focused on distributional properties (particularly tail heavyness), when estimating exchange rates models, has led to the widespread adoption of non-Gaussian conditional error distributions, most commonly the Student-t [38, 39, 40]. The Student-t distribution models more extended tails than the normal distribution and is asymptotically a power law with an exponent $\kappa$ equal to the number of its degrees of freedom. The GARCH model embodies (exponentially decaying) correlations between successive amplitudes (volatility) of the noise but assumes zero correlations between the signs of the successive noise innovations (increments).

Since the probability density function of the Student-t distributed random variable with $\kappa$ degrees of freedom is

$$
t(x, \kappa)=\frac{\Gamma\left(\frac{\kappa+1}{2}\right)}{\Gamma\left(\frac{\kappa}{2}\right) \sqrt{\kappa \pi}}\left(1+\frac{x^{2}}{\kappa}\right)^{-\frac{\kappa+1}{2}},
$$

we have (for $n<\kappa$ )

$$
\left\langle x^{n}\right\rangle= \begin{cases}\kappa^{\frac{n}{2} \frac{\Gamma[(n+1) / 2] \Gamma[(\kappa-n) / 2]}{\Gamma(1 / 2) \Gamma(\kappa / 2)}} & \bmod (\kappa, 2)=0 \\ 0 & \bmod (\kappa, 2)=1\end{cases}
$$

In particular, for $\kappa>2$, we have $\left\langle x^{2}\right\rangle=\kappa /(\kappa-2)$. Hence, to meet Eq. (19),

$$
z=\sqrt{\kappa /(\kappa-2)} \cdot x
$$


whose probability density function is

$$
f(z)=\frac{\Gamma[(\kappa+1) / 2]}{\Gamma[\kappa / 2] \sqrt{\pi(\kappa-2)}}\left(1+\frac{z^{2}}{\kappa-2}\right)^{-\frac{\kappa+1}{2}} .
$$

$z$ tends towards the standardized Gaussian random variable as $\kappa \rightarrow \infty$.

We have generated $\operatorname{GARCH}(1,1)$ noise time series as follows: 1) For a given choice of the parameters and of $\kappa$, generate $t$ random t-distributed random numbers; 2) Obtain $z_{1}, z_{2}, \ldots, z_{t}$ from (22); 3) Generate iteratively $\epsilon_{1}, \epsilon_{2}, \ldots, \epsilon_{t}$ which have zero mean and conditional variance $h_{t}$, using equations (17) and (18); 4) A fixed number of the first data points were discarded in order to remove any sensitivity on the initial condition.

\subsubsection{Numerical simulations}

We used the parametric values quoted in [39]: $\mu=4.38 \times 10^{-4}, \alpha=2.19 \times 10^{-6}, \beta=0.044$, $\gamma=0.922$, and $h_{0}=6.45 \times 10^{-5}$. In Fig. 10, we show four typical simulated $\operatorname{GARCH}(1,1)$ noises: (a) $\kappa=3$, (b) $\kappa=6$, (c) $\kappa=9$, and (d) $\kappa=12$. Local clustering of volatility are clearly visually apparent in the figure.

In order to obtain the significance level of the artificial $\operatorname{GARCH}(1,1)$ noises, we simulated a large number of data sets. The artificial times were unevenly sampled so that each time followed the previous time by a random number between 0 and 1 as in previous simulations. We investigated four types of noises with $\kappa=3,6,9,12$, as typically shown in Fig. 10. For each $\kappa$, we generated 50,000 data sets, each with 100 data points. We then Lomb transformed the 50,000 time series and extracted the maximal Lomb peak height in each periodogram to form a set [2]. The results are shown in Fig. 11. To compare with the independent Gaussian noise, we also simulated 50,000 Gaussian time series and plotted it in the same figure as the open circles.

For a given peak height, the false-alarm probability increases with $\kappa$. This is the effect already documented in Sec. 2. The effect is weak because $\kappa \geq 3$ and the strongest dependence of the false-alarm probability on the power law tail exponent $\kappa$ was shown to occur for smaller exponents. Note that the false-alarm probability of the $\operatorname{GARCH}(1,1)$ process tends to the Gaussian curve $\kappa$ increases. For the largest $\kappa$ investigated, the false-alarm probability is slightly above the Gaussian uncorrelated value but the difference is small. The effect of correlation of the variance of the $\operatorname{GARCH}(1,1)$ process has almost no impact on the false-alarm probability, which is essentially controlled by the non-Gaussian character of the distribution function. This is checked by reshuffling the GARCH noise to eliminate the correlations in volatility. Performing the same analysis, we find the same relation between Lomb peak height and false alarm probability within statistical fluctuations. Since the correlations of the variance are destroyed by reshuffling the $\operatorname{GARCH}(1,1)$ noise while keeping the same heavy tail distribution, we conclude that $\operatorname{GARCH}(1,1)$ correlations have essentially no impact on the false-alarm probability of the detection of periodic components. The slight difference between the false-alarm probabilities reported in Fig. 11 and in Fig. 5 for the uncorrelated power law noise with the same tail exponent can thus be attributed only to the difference in the bulk of their distributions.

\subsection{Fractional Lévy noise}

\subsubsection{Method for fractional Lévy noise synthesis}

We now combine heavy tails and long-range correlations. For this, we study the fractional Lévy motion (fLm) [41, 43, 42], which is analogous to $\mathrm{fBm}$ [20], with the Gaussian distribution of incre- 
ments replaced by a Lévy stable distribution. This corresponds to a moving average with infinite memory over a set of independent random variables having a Lévy distribution. Let us denote $B_{H}^{\alpha}(t)$ for fLm. Then, we have $B_{1 / 2}^{\alpha}(t)=B^{\alpha}(t)$ and $B_{H}^{2}(t)=B_{H}(t)$. Fractional Lévy motion is a self-similar process with self-similar index $2 H / \alpha>0$ [42]:

$$
B_{H}^{\alpha}(a t)_{t \in R} \sim a^{2 H / \alpha} B_{H}^{\alpha}(t)_{t \in R}
$$

for $a>0$. The symbol $\sim$ denotes that the two processes have the same distribution. The heavy-tail behavior of $\mathrm{fLm}$ is [42]

$$
\operatorname{Pr}\left(\left|B_{H}^{\alpha}(t)\right|>x\right) \propto x^{-\alpha / 2 H} .
$$

In order to generate a fractional noise, there are several methods and the simplest one is to use a fractional ARIMA [44]. It is easy to implement the algorithm by replacing the i.i.d. Gaussian noise in the Durbin-Levinson method in Sec. 3 with Lévy noise [44]. In this section, we applied the wavelet method for synthesizing fractional Lévy noise. Accurate synthesis of fBm using wavelet is based on the wavelet analysis of the second order statistics of fractional Brownian processes [31]. Hence, $\mathrm{fBm}$ is generated as a sum of wavelets[32, 34]. Similarly, we can use a wavelet transform of Lévy stable noise and then reconstruct a fractional Lévy noise as in the generation of fBm with wavelet transform [31, 32, 33, 34]. We use the Daubechies wavelet in the simulations. Thus, fractional Lévy noise is the series of increments of the fLm. There are other generating methods, for instance using Fourier transforms, but the errors are more difficult to control.

\subsubsection{Numerical simulations}

We simulated fractional Lévy noises for different Hurst exponents $H$ and heavy-tailness $\alpha$. For each simulation, we fixed the pair $(H, \alpha)$ among $19 \times 40$ possible values: $H$ ranged from 0.05 to 0.95 with spacing 0.05 , while $\alpha$ varied from 0.05 to 2 with step 0.05 . Four typical noises are illustrated in Fig. 12: (a) $H=0.2$ and $\alpha=0.4$, (b) $H=0.4$ and $\alpha=0.8$, (c) $H=0.6$ and $\alpha=1.2$, (d) $H=0.8$ and $\alpha=1.6$.

To qualify the effect of the interplay between correlations and heavy tails in fractional Lévy noises, we introduce a characteristic quantity $h_{p}$ defined as the Lomb peak height corresponding to a certain false-alarm probability $p$. We checked that different choice of $p$ resulted in the same properties of $h_{p}$ reported below, as long as $p$ is not too small. We generated 1,000 time series each with 100 data points for each couple $(H, \alpha)$ of the $19 \times 40$ possible couples.

Fig. 13 shows the dependence of $h_{0.01}$ as a function of $H$ and $\alpha$ in the fractional Lévy noise. $h_{0.01}$ increases both with $H$ and $\alpha$. This is consistent with previous results obtained for each of the ingredients: a large correlation (larger $H$ ) increases the Lomb peaks (and thus false-alarm probability); a large power law exponent was also found to increase the false-alarm probability since this corresponds to weaker fluctuations and thus allows for the random appearance of coherent periodic structures.

\section{Joint-distribution of the frequency and height of the highest peak of the Lomb power spectrum}

Up to now, we have reported the false-alarm probability as a function of the highest Lomb peak, independently of its frequency. In practice, the value of the false-alarm probability as a function of frequency is also an important determinant of the statistical significance of a supposed periodic 
signal. The confidence level of a Lomb peak in a given time series may be increased if one can distinguish the frequency $f^{r}$ corresponding to the highest Lomb peak $h^{r}$ of the real signal from the most probable frequency $f^{m p}$ of a noise determined from synthetic time series. This approach has been used for specific cases in [9, 10].

To address this question, we now investigate the impact of the heavy-tailness of noise distributions and noise correlation on the two-point statistics of $(f, h)$. We study Lévy stable noise and fractional Gaussian noise separately, as this is enough to show the main facts and the numerical calculations become prohibitive for the combined fractional Lévy noises. For each type of noise, we synthesize 50,000 series each with 100 data points. The same algorithms as in Sec. 2.1 are used for the Lévy stable noise and as in Sec. 3.1 for the fractional Gaussian noise.

Fig. 14 shows the probability density distribution $p(f, h)$ of the highest Lomb peak height $h$ and its associated frequency $f$ for the Lévy stable noises, for $\alpha=0.1,1$ and 1.5. In this case of uncorrelated Lévy noise, all frequencies are equiprobable: this is clearly retrieved in the distribution for $\alpha=1.5$. Large fluctuations for the smaller $\alpha$ 's show that the statistical ensemble of these simulations is not sufficiently large to reach the asymptotic independence over the frequencies, as large bursts of probability $p(f, h)$ occur at certain frequencies. The dependence of $p(f, h)$ as a function of $h$ recovers our previous results shown in Fig. 3 .

The probability densities $p(f, h)$ of the highest Lomb peak height $h$ and its associated frequency $f$ for fractional Gaussian noises are shown in Fig. 15, for $H=0.1,0.5$ and 0.9.

- For $H=0.1$ and more generally for $0<H<0.5$ (Fig. 15]a), the fGn is anti-persistent, i.e., the noise tends to reverse its sign, and thus oscillate fast. We should thus expect and do observe periodic components with high frequencies. For instance, in Fig. 8a generated for $H=0.2$, one can observe about 30 "cycles" in the noisy time series, resulting in $f^{m p} \simeq 0.6$.

- For $H=0.5$ (Fig. 15b), the fGn is white-noise and all frequencies should be equivalent, as observed.

- For $H=0.9$ and more generally for $0.5<H<1$ (Fig. 15c), the fGn is persistent, i.e., the noise tends to continue along a trend. We should thus expect and do observe periodic components with very low frequencies. For instance, in Fig. \&d generated for $H=0.9$, one can observe about 2 "cycles" in the noisy time series, resulting in $f^{m p} \simeq 0.02$.

Generally, the number of "cycles" decreases as $H$ increases. Thus, the most probable frequency $f^{m p}$ decreases with increasing $H$, ranging from $\frac{1}{\Delta t}$ to $\frac{N}{3 \triangle t}$.

We have verified in our simulations that the distribution $p(f, h)$ for the $\alpha=2$ Lévy stable noise is identical to the distribution $p(f, h)$ obtained for the fractional Gaussian noise with $H=0.5$, as it should. This provides a confirmation of the validity of the applied algorithms.

\section{Relation with previous works on the detection of log-periodicity}

We now briefly discuss how the simulations presented here shed light on previous announcements of the detection of log-periodicity. To apply the numerical results in this work, there are several issues that should be addressed in advance.

\subsection{Impact of amplitudes of harmonics}

It is natural that the underlying log-periodic function in the noisy signal is not the pure cosine shown in Eq. (3). Then we will see harmonics in the Lomb periodogram. There are several different cases. 
If the amplitude of the fundamental periodic component dominates, i.e. $y_{1} \gg y_{m}$ for all $m>1$, $P_{N}\left(\omega_{1}\right) \gg P_{N}\left(\omega_{m}\right)$. Note that $\omega_{m} / \omega_{1}$ are positive integers. In this situation, we can apply our numerical results directly to determine the significance level of the Lomb peak.

In contrast, if the amplitude(s) of the harmonics is comparable with that of the fundamental periodic component, i.e. $y_{1} \simeq y_{m}$ for some $m>1$, no less than two comparable peaks coexist in the periodogram. In this case, it is more difficult to determining the significance of the peaks, as is implied by Eq. (†).

It is also possible that for some systems there exists at least one harmonic component $m$ such that $y_{1} \ll y_{m}$ leading to $P_{N}\left(\omega_{1}\right) \ll P_{N}\left(\omega_{m}\right)$. Examples, which we have studied recently will be reported elsewhere. They include (1) the log-periodic residues of moments of some self-similar multinomial measures [45]; (2) $q$-derivative [46] of some "Weierstrass-type" functions [47]; and (3) canonically averaged local log-derivative of the moments of energy dissipation rate in threedimensional fully developed turbulence [48].

There are other possible origins which might cause several high peaks in one Lomb periodogram, with low signal-to-noise ratio. If these peaks are disordered, it is hard to conclude that periodic component(s) exist. Meanwhile, high noise will also suppress the fundamental peak and increase harmonic peaks due to the interaction between signal and noise.

For the situations that $y_{1} \gg y_{m}$ fails, all the peaks are not significant if we apply the numerical results directly. However, it is well known that evenly spaced high peaks in the power periodogram is a strong signature of the existence of a fundamental frequency. We can use a least-square fitting to extract the fundamental frequency as proposed in Ref. [45, 48]. This is however beyond the theme of the present paper.

\subsection{Statistics of highest peaks ratio}

In practice, it is always difficult to judge if a given Lomb peak is significant or not, especially when the nature of the noise is unknown. Then, the absolute amplitude of the highest Lomb peak is difficult to interpret and translate into a false-alarm probability and into a confidence level. It is then natural to introduce a measure of relative significance, for instance by using the ratio of the first highest Lomb peak to the second highest peak. Fig. 16 and Fig. 17 show the complementary cumulative distributions of the ratio of the two highest Lomb peaks for Lévy stable noise and fractional Gaussian noise. Again, the number of samples is 50,000 each with 100 data points. The open circles in the two figures correspond to Gaussian noise and the slight discrepancy is caused by the application of different algorithms. The complementary cumulative (false-alarm) probability of a given peak increases with $\alpha$ for Lévy stable noise, which corroborates our previous statistics on the absolute height of Lomb peaks: for small exponent $\alpha$, it is very improbable to observe a large ratio of the two highest Lomb peaks. Thus, a signal showing a large ratio would qualify as highly significant.

The behavior of fGn is non-monotonous. The false-alarm (complementary cumulative) probability decreases with $H$ in the "anti-persistence" regime $0<H \leq 0.5$ and increases with $H$ in the persistence regime $0.5 \leq H<1$. Thus, both mechanisms, which leads to deviations from uncorrelated randomness, lead to an increase the false-alarm probability.

\subsection{Application to real systems}

We now review how the present analysis shed light on our previous works that have used the Lomb analysis to attempt qualifying the presence of log-periodicity in different complex systems. 
Ref. [49] presents Lomb periodograms of the residual of the fit of the distribution of crack lengths by a pure power law. Since the fit is performed with a power law, the natural variable is the logarithm $\ln \ell$ of the crack lengths: a detection of a periodic component in the residual of the distribution of the crack lengths as a function of $\ln \ell$ would qualify log-periodicity. The Lomb periodogram shown in Fig. 14 of [49] for a model of crack growth has a very high peak of 90 with very narrow width and is highly significant, whatever the nature of the noise, Gaussian, Lévy, power law or even with long-range correlations. The Lomb periodogram shown in Fig. 16 of [49] for geological data on the distribution of joint length has two peaks at a level between 45 and 50 which are so close as being barely distinguishable. All the other peaks are much smaller not above 13. These two peaks are very significant, even in the presence of the most unfavorable case of highly persistent noise.

Ref. [50] analyzed experimental data on the time evolution of the number, size and separation of vortices in freely decaying 2-d turbulence to investigate the existence of a discrete time scale invariance, which could reflect that the time-evolution of the merging of vortices is not smooth but punctuated, leading to a preferred scale factor and as a consequence to log-periodic oscillations. Three Lomb periodograms were reported which were averaged over 7-10 realizations of an experiment on freely decaying turbulence: Figs. 5 and 6 of Ref. [50] show the Lomb periodogram of the logarithmic derivative of the number of vortices with respect to time; Figs. 9 and 10 show the Lomb periodogram of the logarithmic derivative of the separation between vortices with respect to time; Figs. 13 and 14 show the Lomb periodogram of the logarithmic derivative of the mean radius of vortices with respect to time. In all cases, the maximum height is no more than about 3.5. The present study suggests that only for heavy-tailed noise with small exponent $\alpha$ or $\kappa$ will each Lomb periodogram achieve a reasonable statistical significance.

In [50], it was argued that the case for log-periodicity was not based on the evidence obtained for each isolated data set but from the collective evidence that the maximum peaks were found for each data set at about the same log-frequency around $4-5$ corresponding to a preferred scaling ratio 1.3. This log-frequency of $4-5$ is much higher than the frequency one would expect from the size of the interval (see Fig. 4 of [50] and the value of the most probable frequency due to noise equal to $1.5 / 1.8=0.8$ ) Thus, statistical significance is argued to be based on the coincidence of three highest peaks of the three Lomb periodograms which occur at frequencies far from the most probable frequencies found in our studies. Taking a number of points around 35 in these data, the signal-to-noise ratio is around $y_{m}^{2} / 2 \sigma_{m}^{2}=0.2$ according to Eq. (đ). This might be the reason for the low Lomb peaks in the periodograms. On the other hand, we cannot find support from the statistics proposed in Sec. 6.2 A further note of caution is necessary: the curves in figures 4, 8, and 12 of Ref. [50] are quite similar to fractional Gaussian noise with $H \approx 0.3$. According to this alternative interpretation, long-range correlations could characterize the this turbulence data, rather than log-periodicity. This example shows the difficulty and complication of determination of the significance level of a given peak in practice.

Ref. [51] reports in its Fig. 1 five Lomb periodograms of five time-dependent ion concentrations in water close to the epicenter of the Kobe earthquake of Jan. 17, 1995 as a function of logfrequency. The two largest peaks are of height 13 and 11. According to the uncorrelated Gaussian benchmark, these two peaks give a false-alarm probability of about or less than $10^{-4}$, suggesting a very strong significance for a genuine log-periodicity. The possible existence of tail-tailed noise reinforce even further this case. However, one cannot exclude the possible existence of some long-range correlation in the ion concentrations, for instance associated with the time-dependent evolution of water permeability. Our present study shows that going from a white-noise spectrum to a $1 / f$ spectrum transform the false-alarm probability from $10^{-4}$ to about $10-20 \%$. 
References [7, 52, 8, 53, 54] report several Lomb periodograms performed to qualify the existence of log-periodicity in the price dynamics of speculative bubbles preceding large financial crashes. The Lomb power spectra are normalized such that the highest peak has a height equal to 1 . Therefore, we cannot establish the corresponding false-alarm probability, using the different models of noises investigated here. It was argued that the correct method, in absence of information on the underlying noise, is the establish the statistical significance on the basis of a large difference between the highest peak and the background. Figure 17 shows that a white-noise null hypothesis requires a ratio larger than about 3 to qualify a genuine log-periodic component at the $99 \%$ confidence level. In the worst and unrealistic scenario where the noise exhibits long-range correlations with persistence, the ratio must be larger. For instance, figure 17 indicates that the ratio must reach 5 if the Hurst exponent is 0.8 or smaller. However, financial time series are known to have very short-ranged and weak correlations in their return and it is thus hard to believe that strong persistence with large Hurst exponents can characterize the statistics of the returns. We stress here that we are not referring to the persistence property of the absolute value of the returns (or volatility) but to that of the returns themselves which is an entirely different problem. Many of the ratios of the larger peak to the second largest one reported in the Lomb periodograms of [7, 52, 8, 53, 54] are of the order or larger than 3 , some reach 5 or more. This signals a high statistical significance. In some instance, the ratio is not large, but it turns out that the second highest peak is associated with a $\log$-frequency harmonic to the log-frequency of the first peak. In this case, the statistics of the ratio does not apply as the existence of harmonics supports the conclusion they are not generated from noise. Hence, the statistical analysis of the ratio of the first highest peak to the second one confirms the confidence of the existence of log-periodicity in finance crashes.

\section{Conclusions}

We have presented statistical tests on the false-alarm probability that a spectral peak in the Lomb periodogram analysis of noisy periodic signal may result from noise. In order to mimic the large variety of noises that may be present in natural and social data, we have investigated several types of noises, including noises with power law distributions and with short and long-range correlations. The false-alarm probability of a periodic component is found to be strongly dependent on the nature of the noise. This underlines the difficulty in concluding unambiguously on the existence of a genuine log-periodicity in noisy signals when the noise properties are not known a priori and are thus difficult to distinguish from the signal. In the light of the statistical tests performed here, we have briefly reviewed the evidences presented in past works on the existence of log-periodicity in turbulence, earthquake and financial data. Our present study weakens the credibility for 2D-freely decaying turbulence and strengthens it for fracture, for the ion-signature precursors to the Kobe earthquake and for financial markets.

We hope that the present work will help in assessing more reliably the existence of periodicity in noisy complex time series and will provide useful guidelines to test new data sets.

Acknowledgments: We thank T. Huillet and J.-F. Muzy for discussions and D. Stauffer for a careful reading of the manuscript. This work was partially supported by NSF-DMR99-71475 and the James S. Mc Donnell Foundation 21st century scientist award/studying complex system.

\section{References}


[1] Press, W., S. Teukolsky, W. Vetterling and B. Flannery, Numerical Recipes in FORTRAN: The Art of Scientific Computing (Cambridge University, Cambridge, 1996).

[2] Scargle, J.D., Study in astronomical time series analysis. II. Statistical aspects of spectral analysis of unevenly spaced data, Astrophysical Journal 263, 835-853 (1982).

[3] Horne, J.H. and S.L. Baliunas, A prescription for period analysis of unevenly sampled time series, Astrophysical Journal 302, 757-763 (1986).

[4] Press, W.H. and G.B. Rybicki, Fast algorithm for spectral analysis of unevenly sampled data, Astrophysical Journal 338, 277-280 (1989).

[5] Sornette, D., Discrete scale invariance and complex dimensions, Phys. Rep. 297, 239-270 (1998).

[6] Johansen, A. and D. Sornette, Modeling the stock market prior to large crashes, Eur. Phys. J. B 9, 167-174 (1999).

[7] Johansen, A. and D. Sornette, Critical crashes, Risk 12, 91-94 (1999).

[8] Johansen, A., D. Sornette and O. Ledoit, Predicting financial crashes using discrete scale invariance, Journal of Risk 1, 5-32 (1999).

[9] Huang, Y., A. Johansen, M.W. Lee, H. Saleur and D. Sornette, Artifactual log-periodicity in finite-size data: relevance for earthquake aftershocks, J. Geophys. Res. 105, 25451-25471 (2000).

[10] Huang, Y., H. Saleur and D. Sornette, Reexamination of log-periodicity observed in the seismic precursors of the 1989 Loma Prieta earthquake, J. Geophysical Research 105, 28111-28123 (2000).

[11] Sornette, D., A. Johansen, A. Arneodo, J.-F. Muzy and H. Saleur, Complex fractal dimensions describe the internal hierarchical structure of DLA, Phys. Rev. Lett. 76, 251-254 (1996).

[12] Lévy, P., Théorie de L'addition des Variables Aléatoires (Gauthier-Vilars, Paris, 1937).

[13] Painter, S., G. Beresfor and L. Paterson, On the distribution of seismic reflection coefficients and seismic amplitudes, Geophysics 60, 1187-1194 (1995).

[14] Samorodnisky. G. and M.S. Taqqu, Stable, Non-Gaussian Random Processes (Chapman and Hall, NY, 1994).

[15] Harmantzis, F.C., D. Hatzinakos and I. Katzela, Tail probabilities for multiplexing of fractional $\alpha$-stable broadband traffic, IEEE ICC'01, Helsinki, Finland, (2001) ${ }^{\text {t }}$

[16] Feller, W., An Introduction to Probability Theory and Its Applications (Willey, New York, 1971).

[17] Mantegna, R.N. and H.E. Stanley, Stochastic process with ultraslow convergence to a Gaussian: The truncated Lévy flight, Phys. Rev. Lett. 73, 2946-2949 (1994).

\footnotetext{
${ }^{4}$ Available at http://www.comm.toronto.edu/ fotios/papers/papers.htm
} 
[18] Chambers, J.M., C.L. Mallows and B.W. Stuck, A method for simulating stable random variables, J. Am. Statist. Assoc. 71, 340-344 (1976).

[19] McCulloch, J.H., On the parametrization of the $\alpha$-stable distributions, Bull. London Math. Soc. 28, 651-655 (1996).

[20] Mandelbrot, B.B. and J.M. Van Vess, Fractional Brownian motions, fractional noises and applications, SIAM Review 10, 422-438 (1968).

[21] Mandelbrot, B.B., The Fractal Geometry of Nature (Freeman and Company, New York, 1982).

[22] Lau, W.C., A. Erramilli, J. Wang and W. Willinger, Self-similar trafic generation: The random midpoint displacement algorithm and its properties, IEEE Int. Conf. on Comm., 466-472 (1995).

[23] Norros, I., P. Mannersalo and J.L. Wang, Simulation of fractional Brownian motion with conditionalized random midpoint displacement, Adv. Perf. Anal. 2, 77-101 (1999).

[24] Lundhal, T., W.J. Ohley, S.M. Kay and R. Siffert, Fractional brownian motion: maximum likelihood estimator and its application to image texture, IEEE Trans. Medical Imaging MI-5, 152-161 (1986).

[25] Brockwell, P.J. and R.A. Davis, Time Series: Theory and Method (New York: Springer-verlag, 1991).

[26] Taqqu, M.S., V. Teverovsky and W. Willinger, Estimators for long-range dependence: An empirical study, Fractals 3, 785-798 (1995).

[27] Barnsley, M.F., R.L. Devaney, B.B. Mandelbrot, H.O. Peitgen, D. Saupe and R.F. Voss, The science of fractal images (Springer-Verlag, Berlin, 1988).

[28] Flandrin, P., On the spectrum of fractional Brownian motions, IEEE Transactions on Information Theory 35, 197-199 (1989).

[29] Dietrich, C.R. and G.N. Newsam, Fast and exact simulation of stationary Gaussian processes through circulant embedding of the covariance matrix, SIAM Journal on Scientific Computing 18, 1088-1107 (1997).

[30] Crouse, M.S. and R.G. Baraniuk, Fast, exact synthesis of Gaussian and nonGaussian longrange-dependent processes, IEEE Transactions on Information Theory, submitted (1999)

[31] Flandrin, P., Wavelet analysis and synthesis of fractional Brownian motion, IEEE Transactions on Information Theory 38, 910-917 (1992).

[32] Stoksik, M.A., R.C. Lane and D.T. Nguyen, Accurate synthesis of fractional Brownian motion using wavelets, Electronics Letters 30, 383-384 (1994).

[33] Heneghan, C., S.B. Lowen and M.C. Teich, Two-dimensional fractional Brownian motion: wavelet analysis and synthesis, Proceedings of the IEEE Southwest Symposium on Image Analysis and Interpretation, 213-217 (1996).

[34] Hu, G., S.H. Zhu, and B. Xie, Wavelet synthesis of fractional Brownian motion, WCCC-ICSP 2000, 1, 349-352 (2000).

\footnotetext{
${ }^{5}$ Available at http://www-dsp.rice.edu/publications/pub/fftMC.ps.gz
} 
[35] Jennane, R., R. Harba and G. Jacquet, Quality of synthesis and analysis methods for fractional Brownian motion, IEEE Digital Signal Processing Workshop Proceedings, 307-310 (1996).

[36] Engle, R.F., Autoregressive conditional heteroscedasticity with estimates of the variance of United Kingdom inflation, Econometrica 50, 987-1007 (1982).

[37] Bollerslev, T., Generalized autoregressive conditional heteroskedasticity, Journal of Econometrics 3, 307-327 (1986).

[38] Bollerslev, T., A Conditional heteroskedastic time series model for speculative price and rate of return, Review of Economics and Statistics 9, 542-5427 (1987).

[39] Johansen, A., O. Ledoit and D. Sornette, Crashes as critical points, International Journal of Theoretical and Applied Finance 3, 219-255 (2000).

[40] Wang, K.L., C. Fawson, C.B. Barrett and J.B. McDonald, A flexible parametric GARCH model with an application to exchange rates, Journal of Applied Econometrics, to appear.

[41] Taqqu, M.S., Random processes with long-range dependence and high variability, J. Geophys. Res. D 92, 9683-9686 (1987).

[42] Huillet, T., Fractional Lévy motions and related processes, J. Phys. A: Math. Gen. 32, $7225-$ 7248 (1999).

[43] Kogon, S.M. and D.G. Manolakis, Signal modeling with self-similar $\alpha$-stable processes: the fractional Lévy stable motion model, IEEE Transactions on Signal Processing 44, 1006-1010 (1996).

[44] Taqqu, M.S. and P. Kokoszka, Can one use the Durbin-Levinson algorithm to generate infinite variance fractional ARIMA time series?, Journal of Time Series Analysis 22, 317-337 (2001).

[45] Zhou W.-X. and D. Sornette, Discrete scale invariance in fractals and multifractal measures, 2001, in preparation.

[46] Erzan, A., Finite $q$-differences and the discrete renormalization group, Phys. Lett. A 225, 235-238 (1997).

[47] Gluzman, S. and D. Sornette, Log-periodic route to fractal functions, Phys. Rev. E, preprint at http://arXiv.org/abs/cond-mat/0106316.

[48] Zhou W.-X. and D. Sornette, Discrete scale invariance of dissipation rate of kinetic energy in three-dimensional fully developed turbulence, 2001, in preparation.

[49] Huang, Y., G. Ouillon, H. Saleur and D. Sornette, Spontaneous generation of discrete scale invariance in growth models, Physical Review E 55, 6433-6447 (1997).

[50] Johansen, A., D. Sornette and A.E. Hansen, Punctuated vortex coalescence and discrete scale invariance in two-dimensional turbulence, Physica D 138, 302-315 (2000).

[51] Johansen, A., H. Saleur and D. Sornette, New evidence of earthquake precursory phenomena in the 17 Jan. 1995 Kobe earthquake, Japan, Eur. Phys. J. B 15, 551-555 (2000). 
[52] Johansen, A. and D. Sornette, Financial anti-bubbles: log-periodicity in Gold and Nikkei collapses, Int. J. Mod. Phys. C 10(4), 563-575 (1999).

[53] Johansen, A. and D. Sornette, Bubbles and anti-bubbles in Latin-American, Asian and Western stock markets: An empirical study, in press in International Journal of Theoretical and Applied Finance (e-print at http://xxx.lanl.gov/abs/cond-mat/9907270).

[54] Sornette, D. and A. Johansen, Significance of log-periodic precursors to financial crashes, Quantitative Finance 1 (4), 452-471 (2001). 
(a)

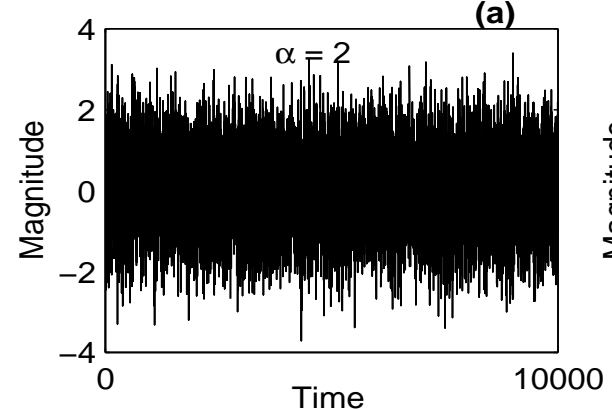

(c)

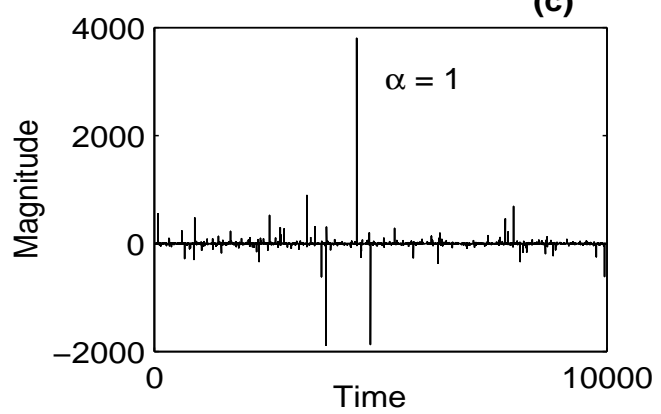

(b)

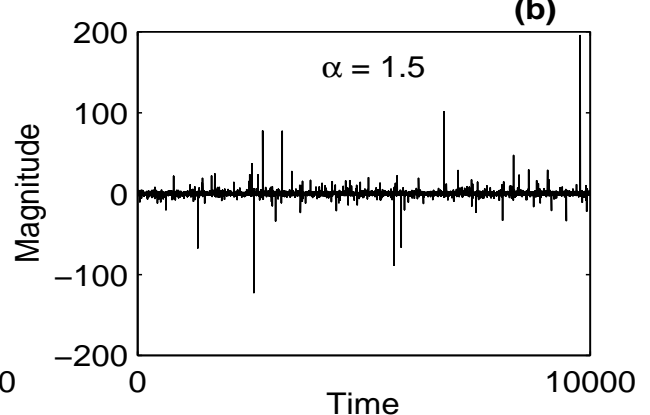

(d)

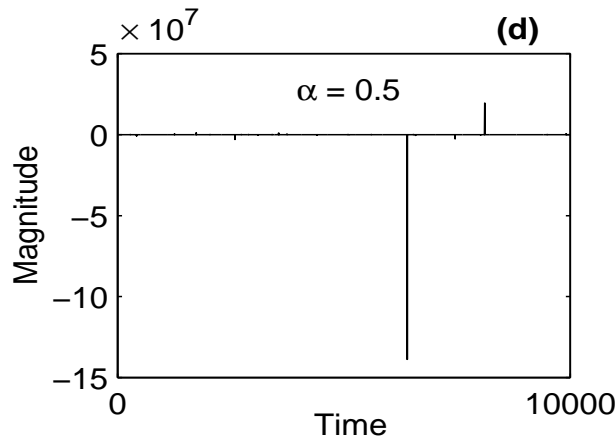

Figure 1: Surrogate Lévy stable noises each with 10,000 data points for: (a) $\alpha=2$; (b) $\alpha=1.5$; (c) $\alpha=1$; and (d) $\alpha=0.5$. Here, $\lambda=0, \mu=0$ and $\sigma=1 / \sqrt{2}$. Note the difference in the vertical scales of the four panels. 
(a)

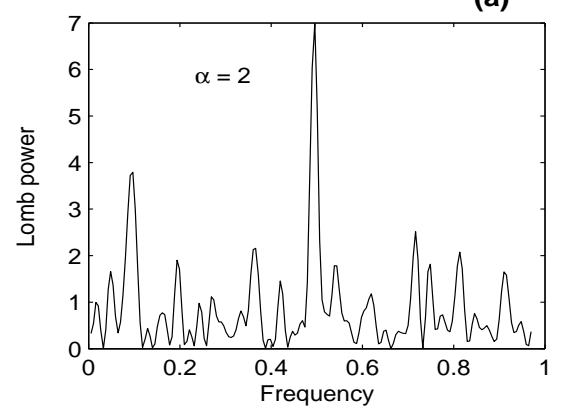

(c)

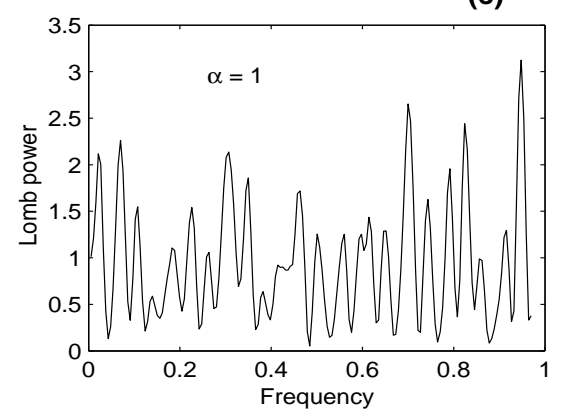

(b)

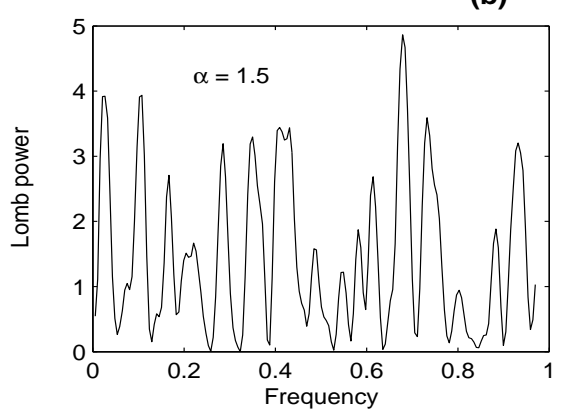

(d)

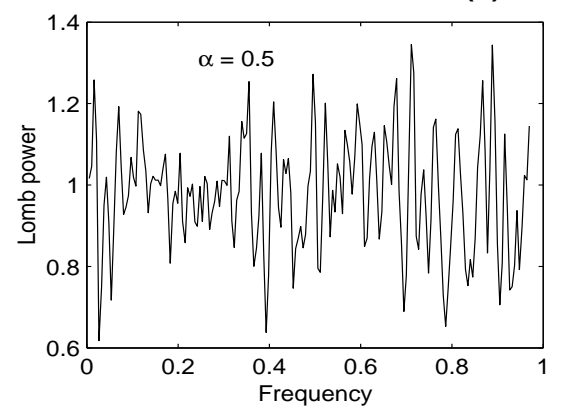

Figure 2: The four Lomb periodograms corresponding to the four cases shown in Fig. 11 1 but with time series of only 100 points. 


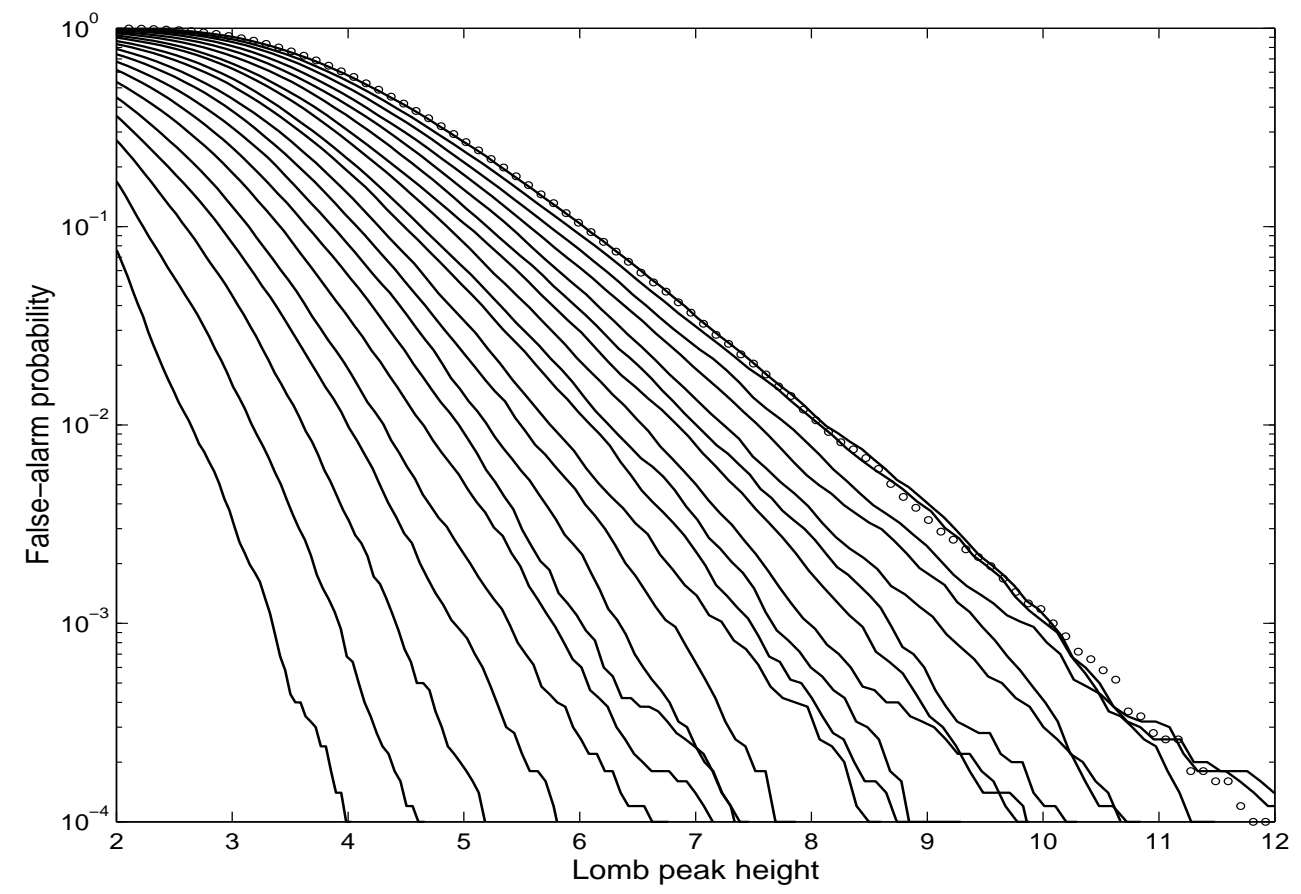

Figure 3: False-alarm probability of the surrogate Lévy noises for $\alpha$ ranging from 0.1 to 2 with step 0.1 from left to right. For a given Lomb peak height, the false-alarm probability increases with $\alpha$. 

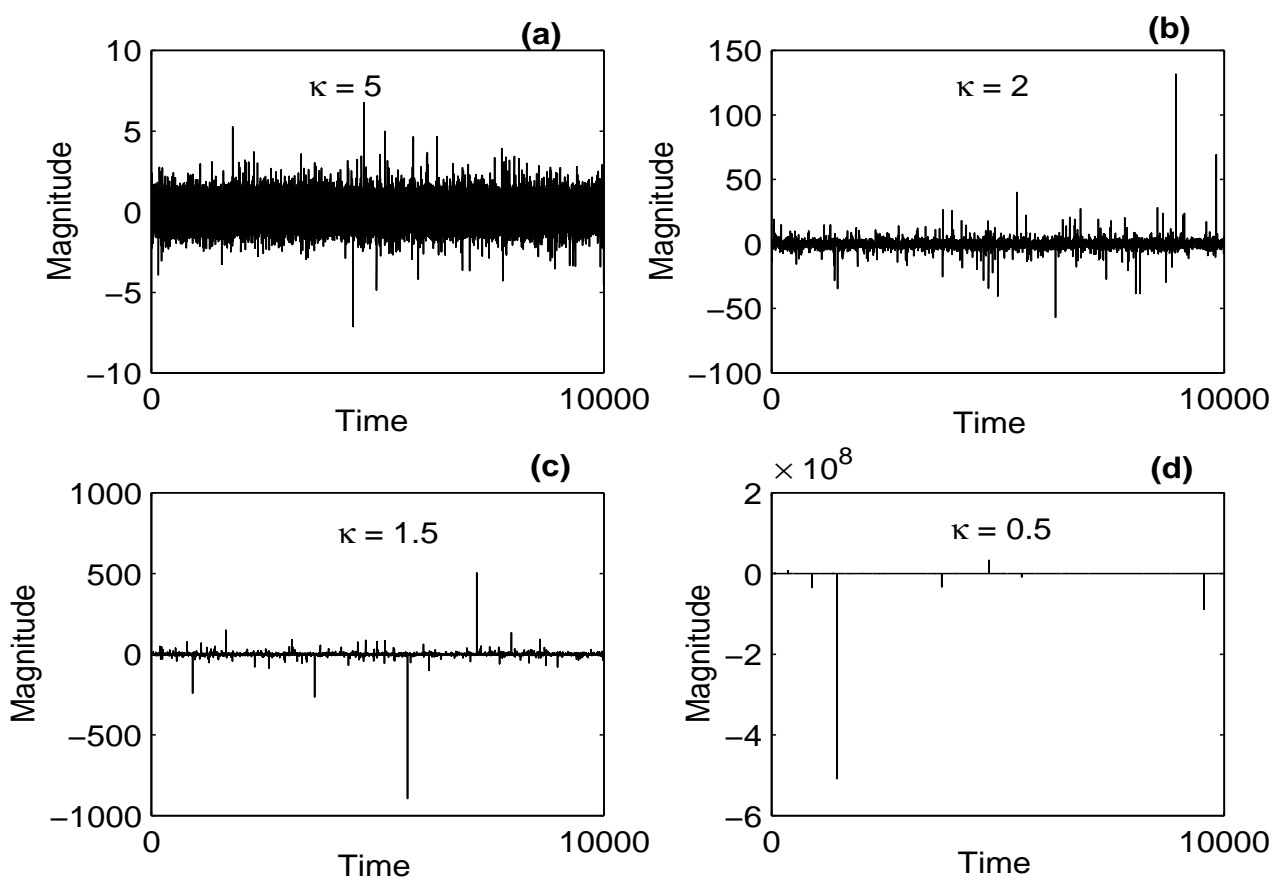

Figure 4: Surrogate symmetrical power-law noises each with 10,000 data points for: (a) $\kappa=5$; (b) $\kappa=2$; (c) $\kappa=1.5$; and (d) $\kappa=0.5$. Note the difference in the vertical scales of the four panels. 


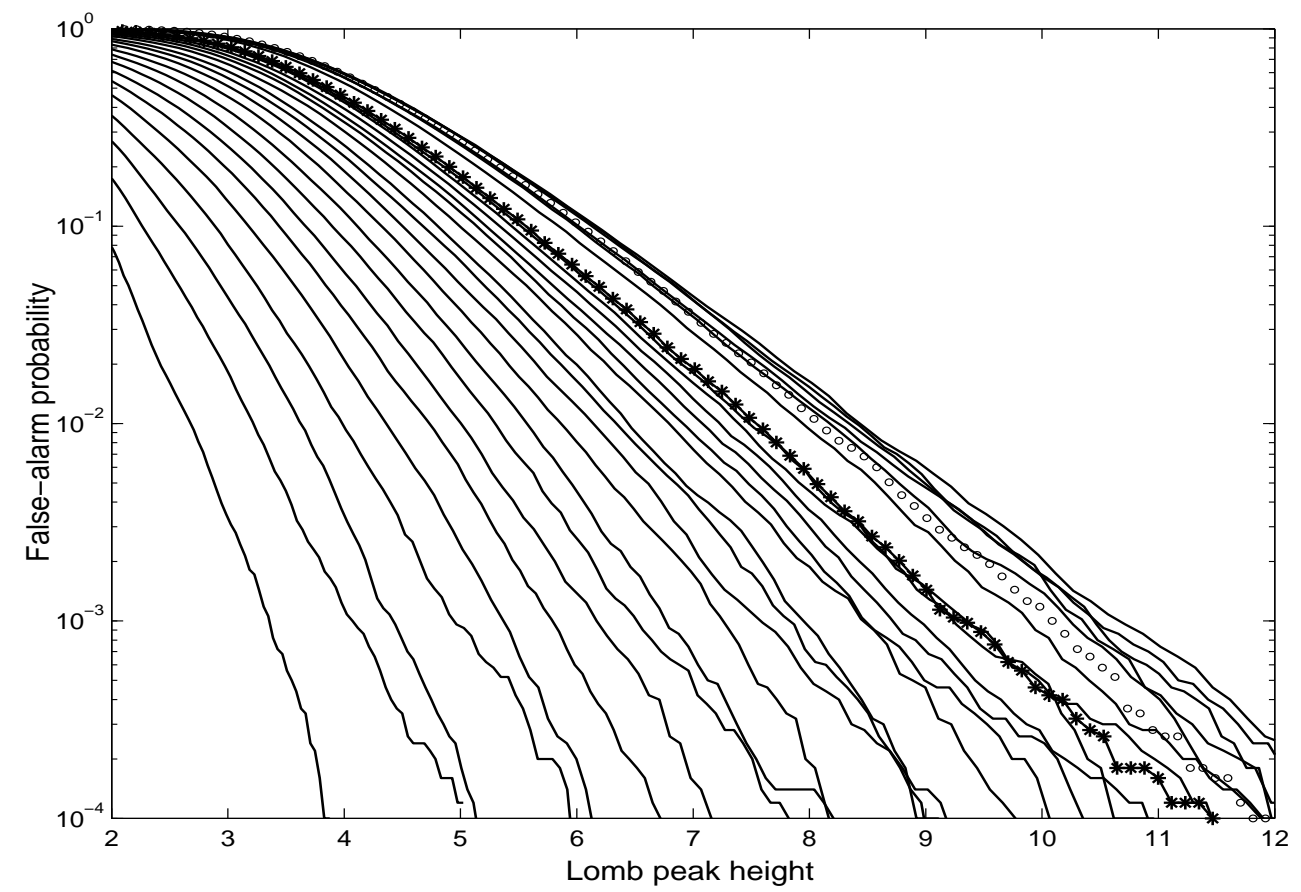

Figure 5: False-alarm probability of the surrogate power-law tailed noise for $\kappa$ ranging from from 0.1 to 2 with spacing 0.1 , from 2.5 to 4 with spacing 0.5 , and for 5 and 6 , from left to right. For a given Lomb peak height, the false-alarm probability increases with $\kappa$. The line marked with stars is for $\kappa=2$ while the circles correspond to the Gaussian case. 


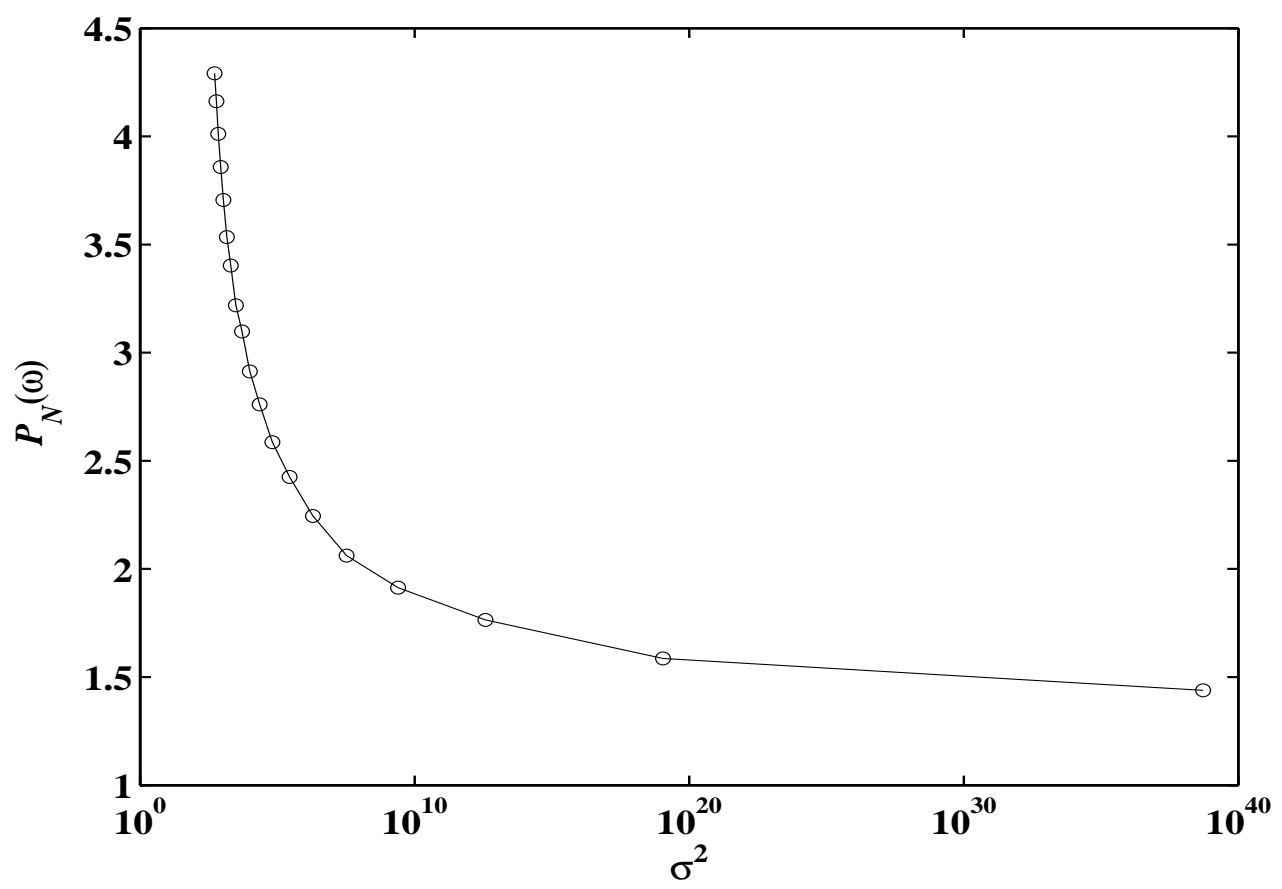

Figure 6: Dependence of the maximal Lomb peak $P_{N}(\omega)$ averaged over 50,000 realizations of 100 points as a function of $\tilde{\sigma}^{2}$ given by expression (13).

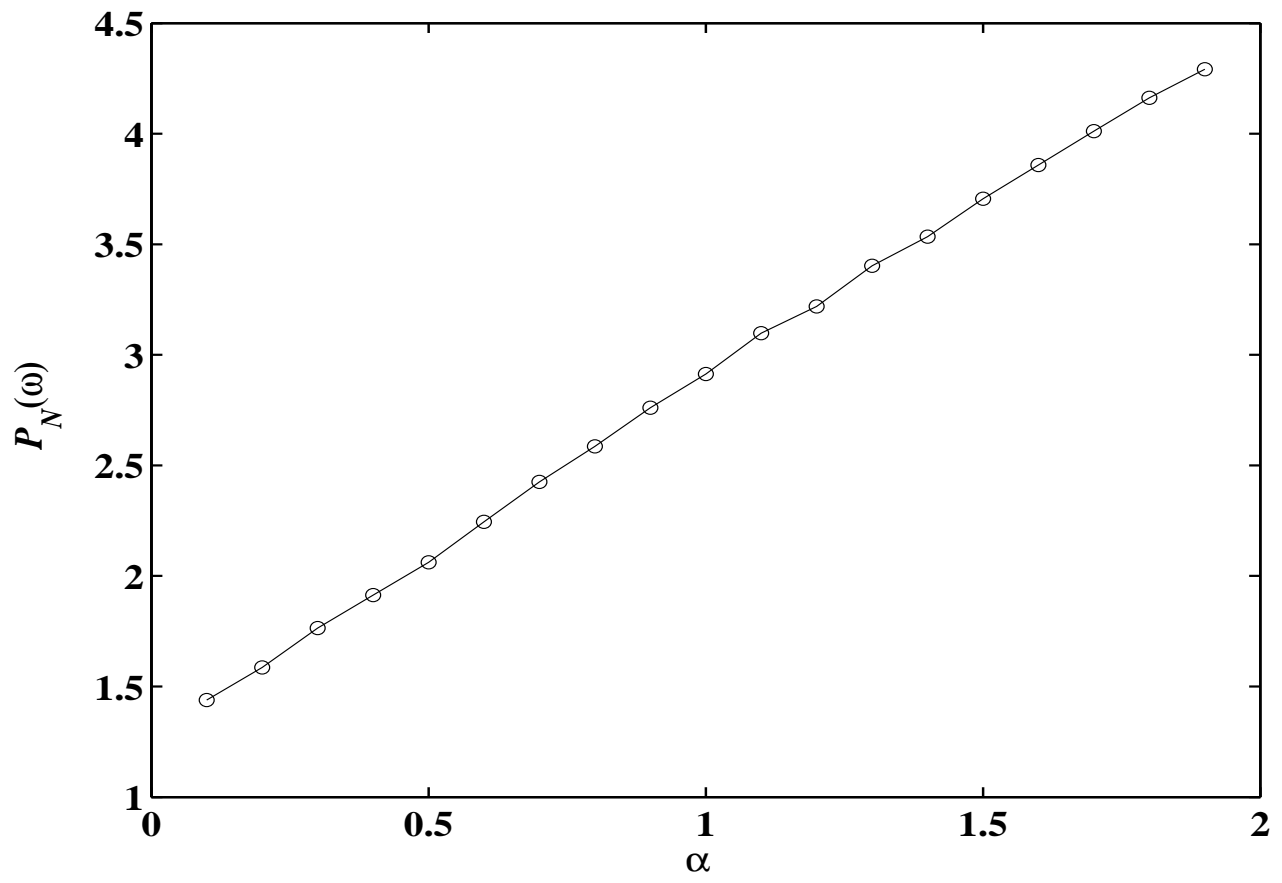

Figure 7: Dependence of $P_{N}(\omega)$ as a function of the power law exponent $\alpha$. 
(a)

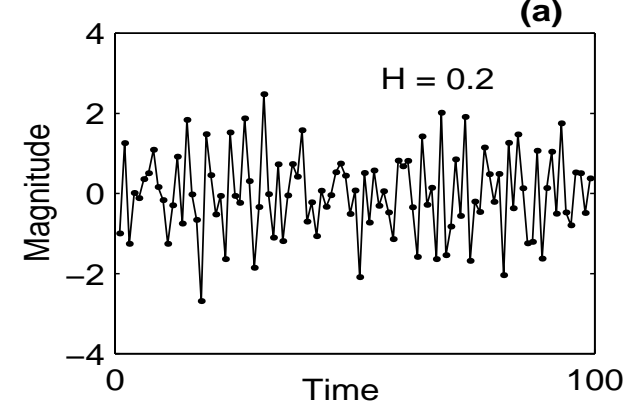

(c)

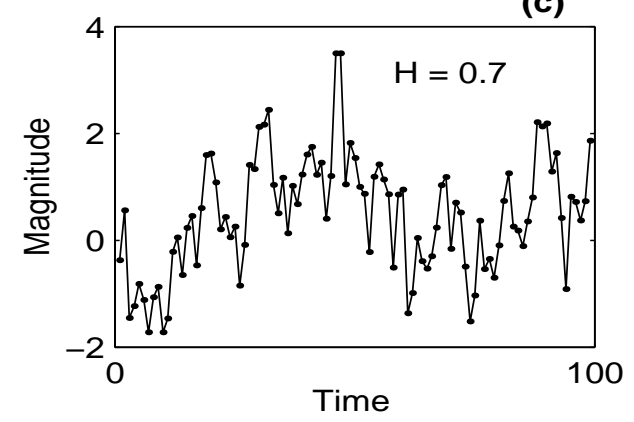

(b)

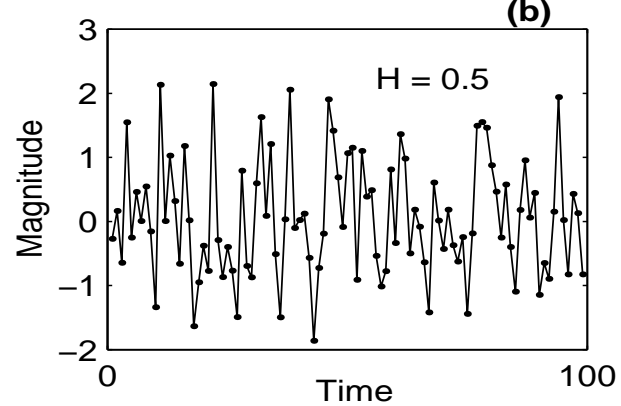

(d)

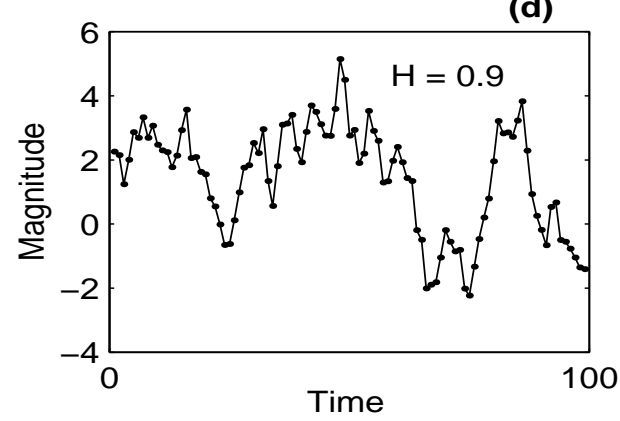

Figure 8: Surrogate fractional Gaussian noises using the Durbin-Levinson algorithm. Each noise has 100 data points whose Hurst indexes are: (a) $H=0.2$, (b) $H=0.5$, (c) $H=0.7$, and (d) $H=0.9$. With the increase of $H$, the fGn exhibits stronger regularity. 


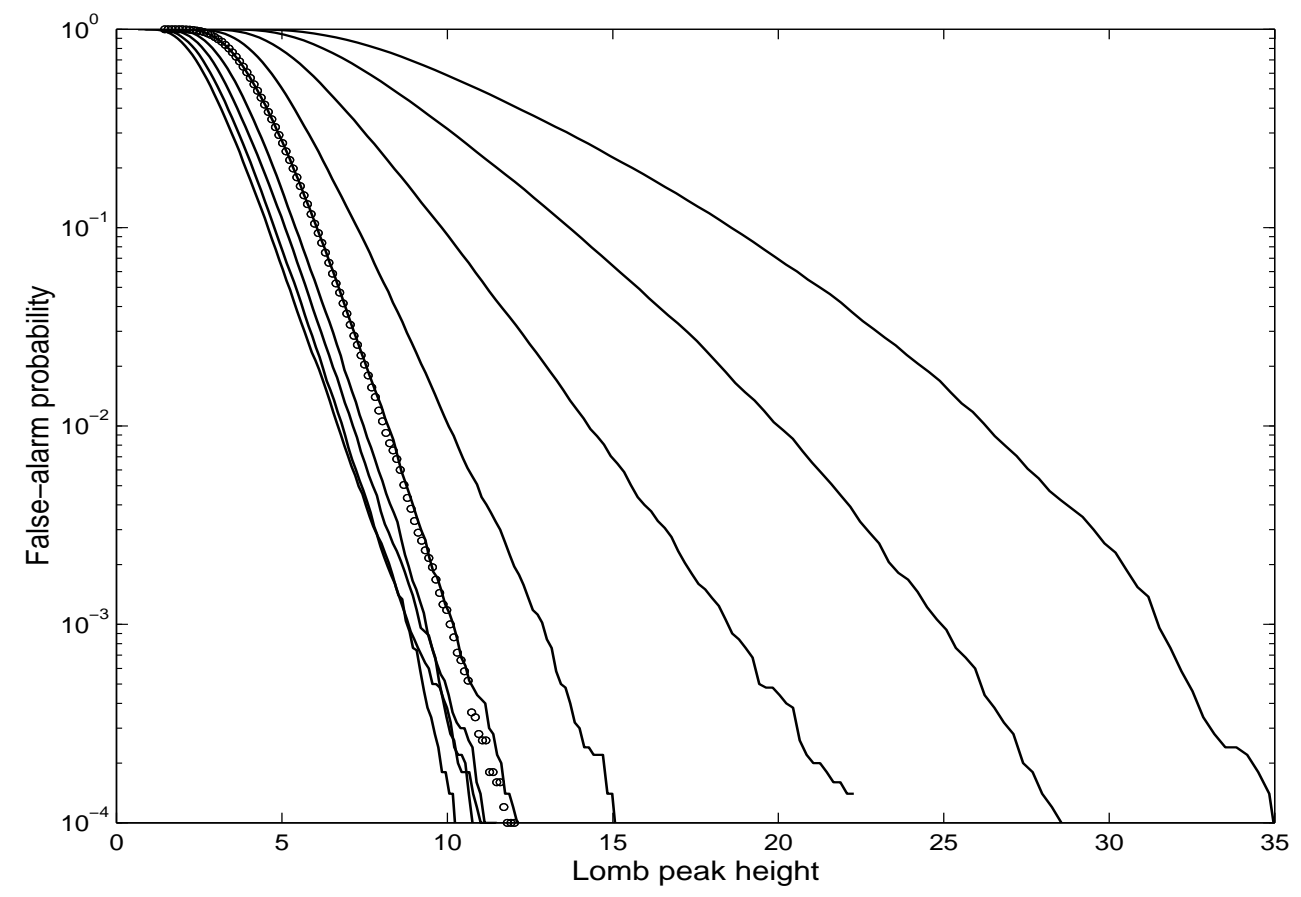

Figure 9: The relationship between the Lomb peak height and the significant level of 50,000 synthetic noises with 100 data points. The Hurst index increases from 0.1 to 0.9 with spacing 0.1 from left to right. 

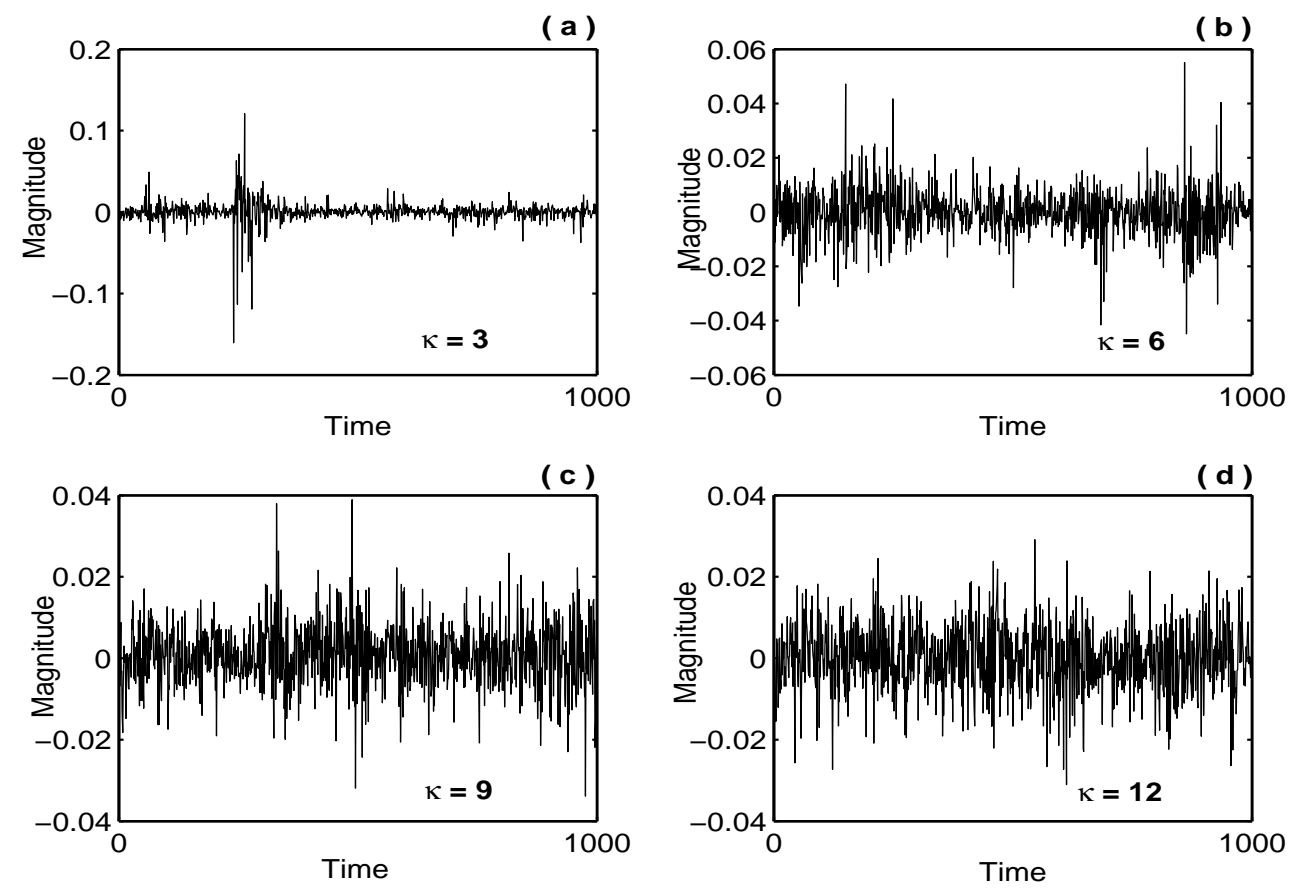

Figure 10: Surrogate $\operatorname{GARCH}(1,1)$ noises using Eqs. (17) with: (a) $\kappa=3$, (b) $\kappa=6$, (c) $\kappa=9$, and (d) $\kappa=12$. 


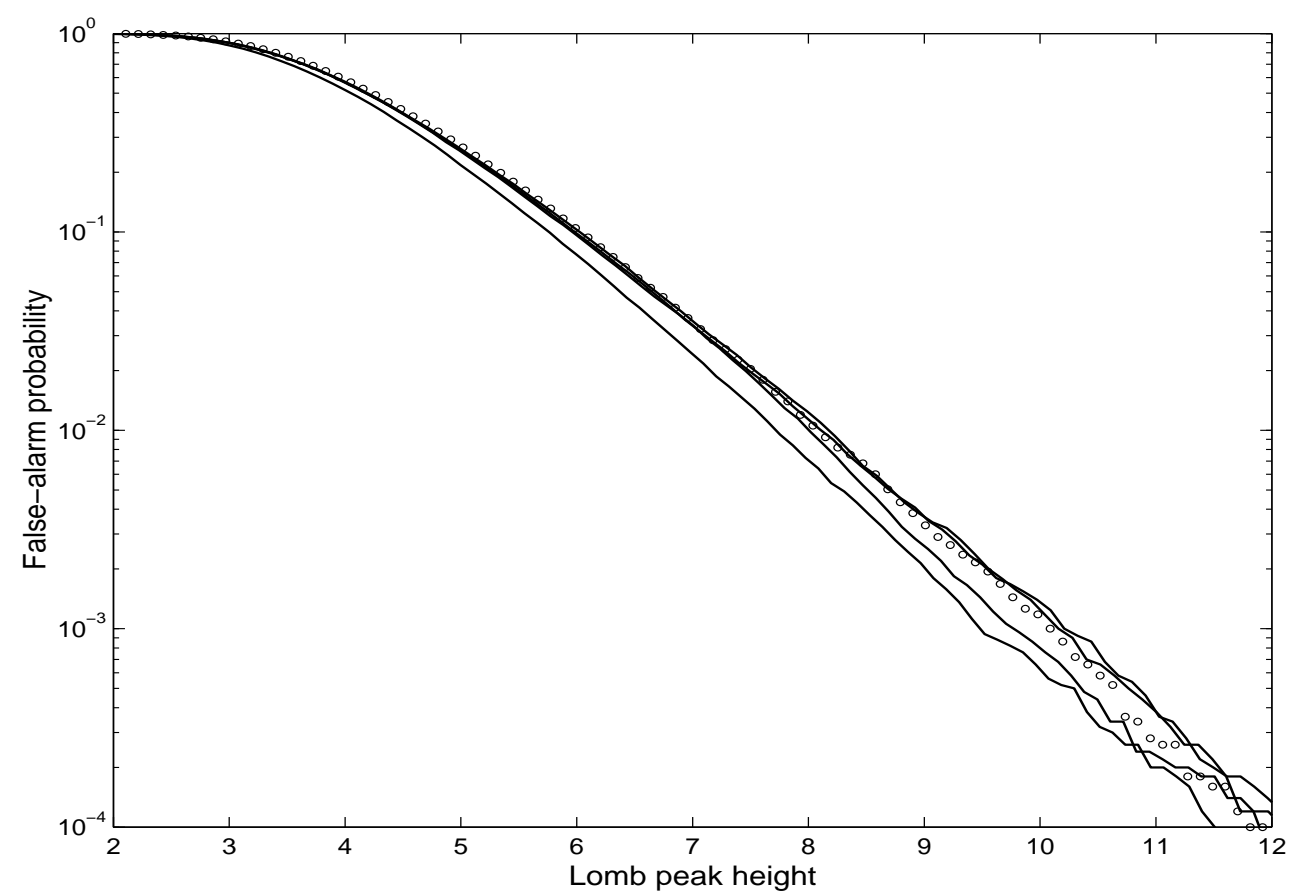

Figure 11: Significance levels of the surrogate $\operatorname{GARCH}(1,1)$ data for $\kappa=3,6,9,12$ and comparison with the independent Gaussian noise.
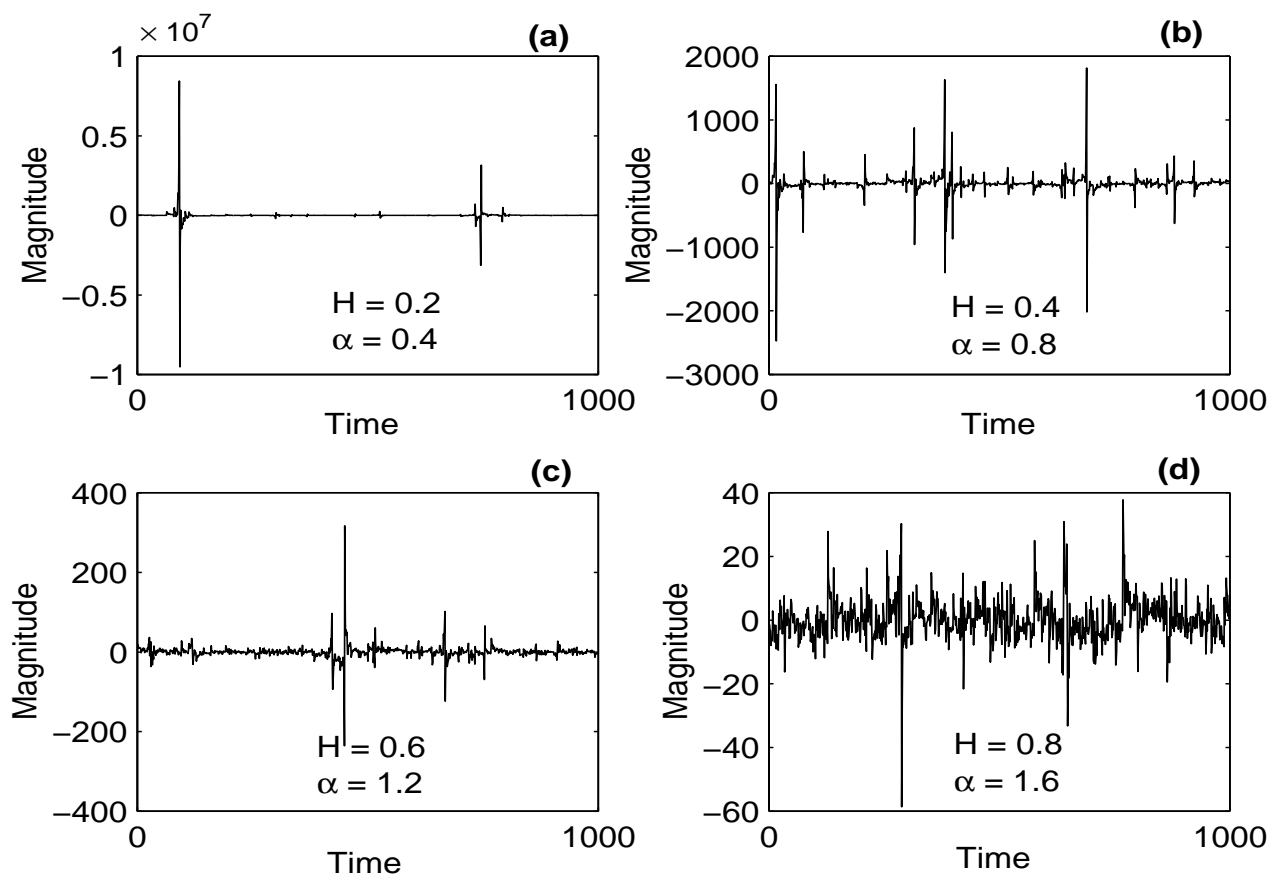

Figure 12: Fractional Lévy noises generated with the wavelet method using Daubechies wavelets. 


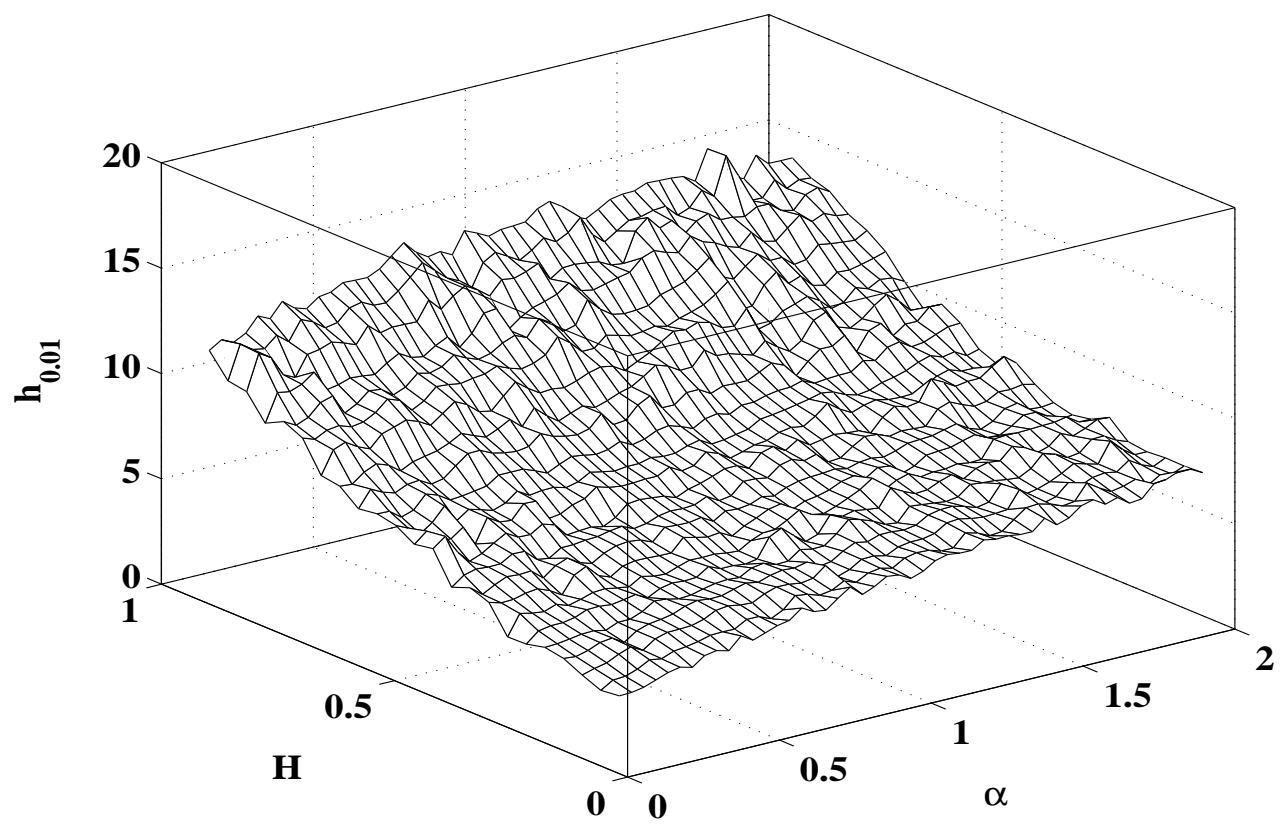

Figure 13: Dependence of $h_{0.01}$ on $H$ and $\alpha$ in fractional lévy noise. $h_{0.01}$ increases with $H$ (respectively $\alpha$ ) for fixed $\alpha$ (respectively $H$ ). Two-dimensional interpolations were carried out to smoothen the surface. The residual oscillations stem from statistical fluctuations. 

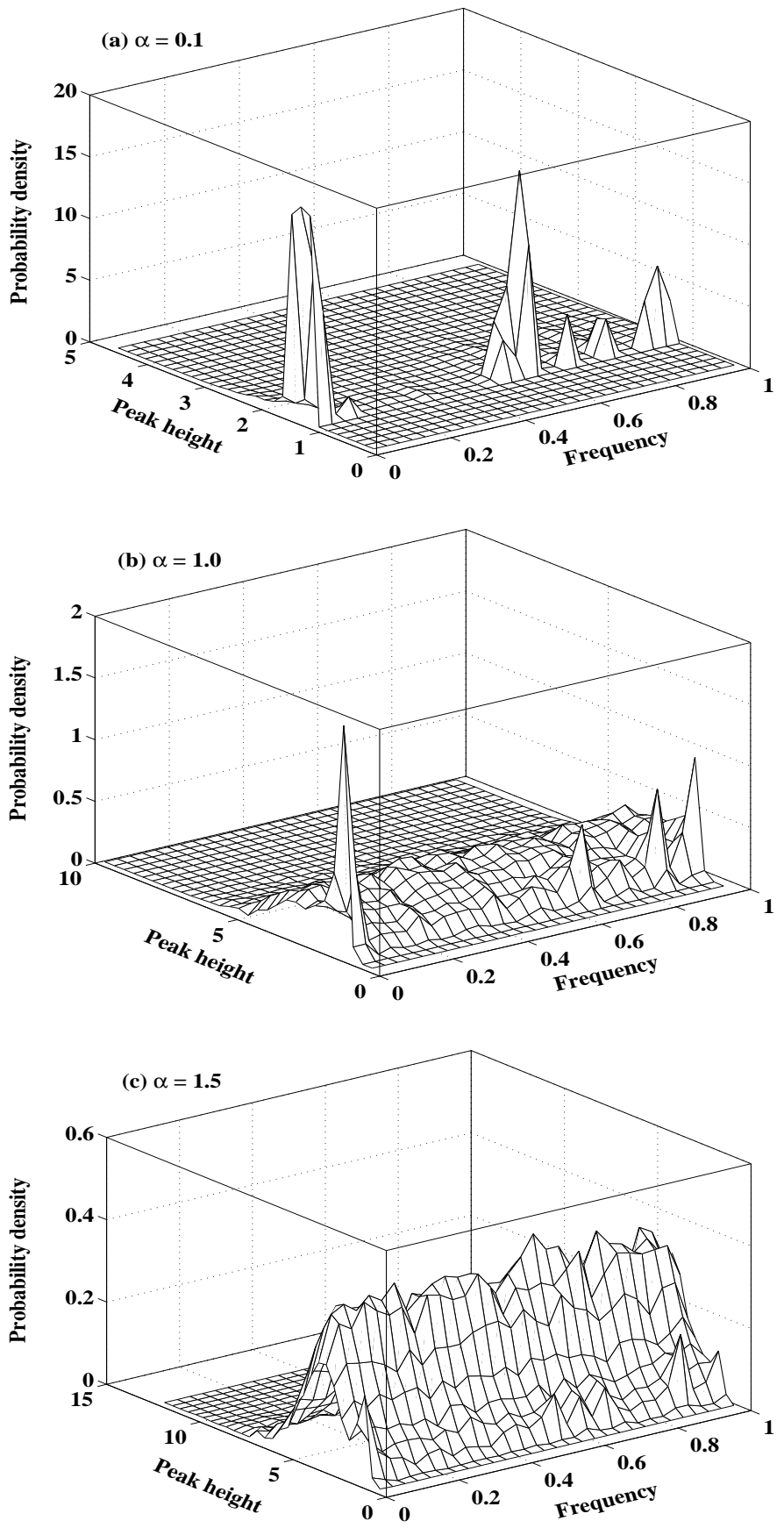

Figure 14: Probability density $p(f, h)$ of the highest Lomb peak height $h$ and its associated frequency $f$ for Lévy stable noises: (a) $\alpha=0.1$, (b) $\alpha=1$, and (c) $\alpha=1.5$. 

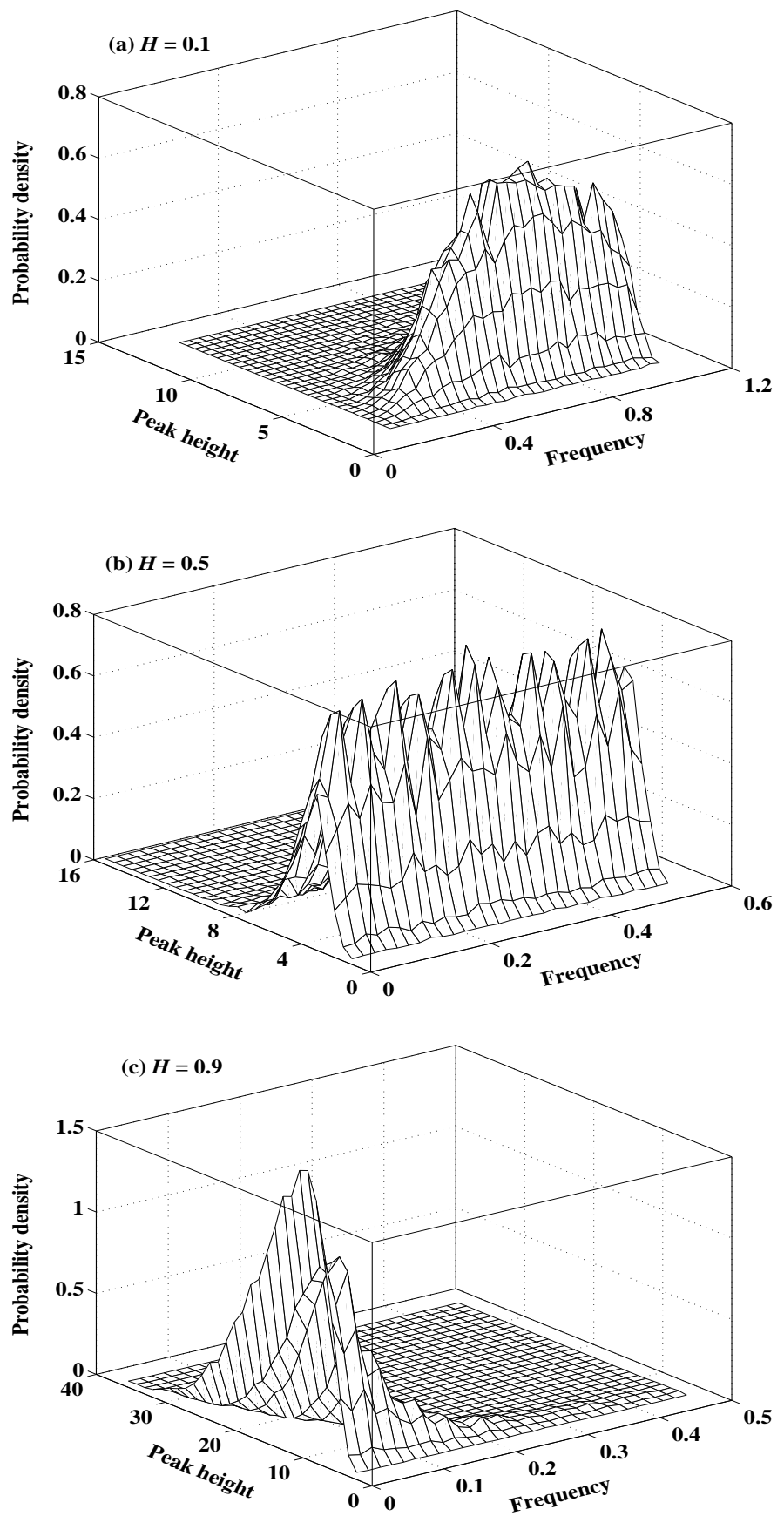

Figure 15: Three types of probability density $p(f, h)$ of the highest peak for fractional Gaussian noise: (a) $H=0.1$, (b) $H=0.5$, and (c) $H=0.9$. 


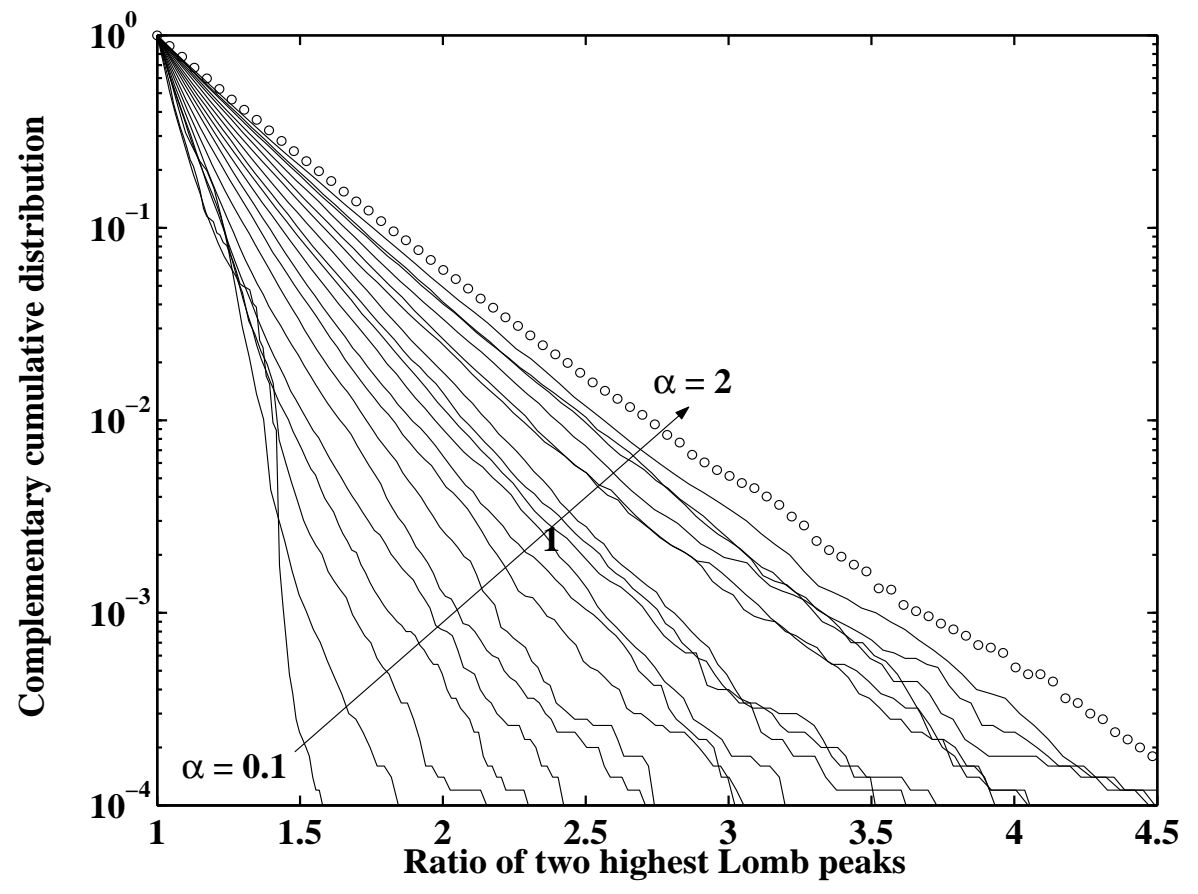

Figure 16: Complementary cumulative distribution of the ratio of the highest Lomb peaks for Lévy stable noise with $\alpha$ varying from 0.1 to 2 as indicated by the arrow. The open circles corresponds to $\alpha=2$. 


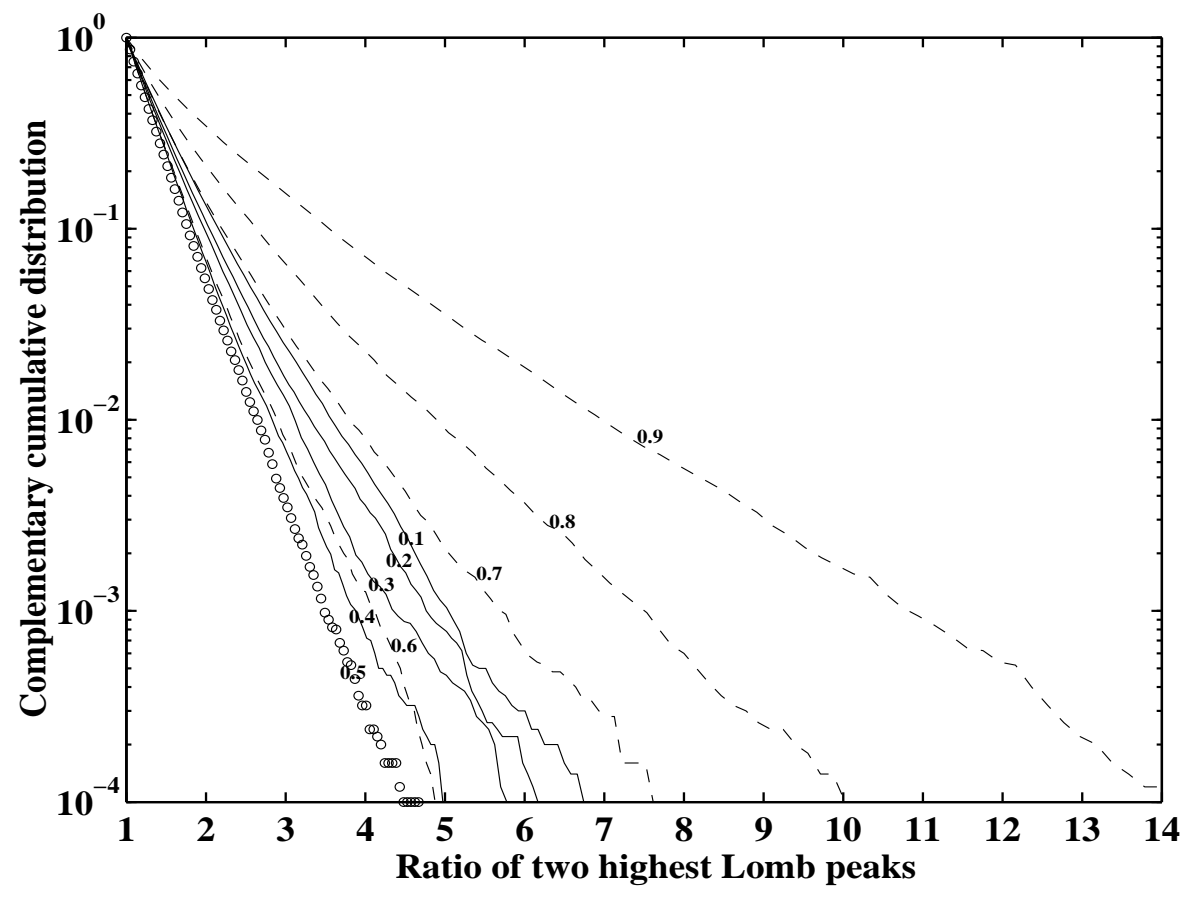

Figure 17: Complementary cumulative (false-alarm) distribution of the ratio of the highest Lomb peaks for fractional Gaussian noise with $H$ varying from 0.1 to 0.9 . The open circles, solid lines and dashed lines correspond respectively to $H=0.5,0<H<0.5$ and $0.5<H<1$. 